\title{
Intensity Based Spatial Soundfield Reproduction Using an Irregular Loudspeaker Array
}

\author{
Huanyu Zuo, Student Member, IEEE, Prasanga N. Samarasinghe, Member, IEEE, \\ and Thushara D. Abhayapala, Senior Member, IEEE
}

\begin{abstract}
Sound intensity is an acoustic quantity closely linked with human perception of sound location, and it can be controlled to create a high level of realism to humans in soundfield reproduction systems. In this paper, we present an intensity matching technique to optimally reproduce sound intensity over a continuous spatial region using an irregular loudspeaker array. This avoids several known limitations in the previous works on intensity based soundfield reproduction, such as a single sweet spot for the listener and a regular loudspeaker geometry that is difficult to implement in real-world applications. In contrast to the previous works, the new technique uses a cost function we built to optimize sound intensity over space by exploiting spatial sound intensity distributions. The spatial sound intensity distribution is represented by spherical harmonic coefficients of sound pressure, which are widely used to describe a spatial soundfield. Compared to the conventional spatial soundfield reproduction method of pressure matching in the spherical harmonic domain and the HOA $\max -r_{E}$ decoding method optimizing sound intensity at a single position, we show that the intensity matching technique has better overall performance with two different irregular loudspeaker layouts through simulations. The impact of microphone noise on reproduction performance is also assessed. Finally, we carry out perceptual localization experiments to validate the proposed method.
\end{abstract}

Index Terms-Sound intensity, spatial soundfield reproduction, irregular loudspeaker array, spherical harmonics, listening test.

\section{INTRODUCTION}

$\mathbf{S}$ INCE the stereophonic sound was introduced, the demand for immersive audio has increased steadily. To cater to the demand, a number of loudspeakers are deployed in home entertainment systems (e.g., 5.1/7.1 systems) or modern cinemas (e.g., Dolby Atmos and DTS:X), where the aim is to create an immersive sound over a spatial region so that the listener inside the region can experience a realistic but virtual replication of the original sound. In this paper, we will present a practical spatial soundfield reproduction system that can recreate a good 3D sound direction perception over space using a loudspeaker array easily implemented in real-world applications.

There have been various studies of soundfield reproduction and they have evolved in two directions: physically motivated techniques and perceptually motivated techniques [1]. Physically motivated techniques aim to reproduce an accurate physical approximation of a sound field. A notable example is Wave Filed Synthesis (WFS) [2]-[4], which reproduces

The authors are with the Research School of Engineering, College of Engineering and Computer Science, The Australian National University, Canberra, ACT 2601, Australia (e-mail: huanyu.zuo@anu.edu.au; prasanga.samarasinghe@anu.edu.au; thushara.abhayapala@anu.edu.au).

This work is supported by ARC Discovery Grant No. DP180102375 and the China Scholarship Council with the Australian National University. desired sound pressure based on Huygens principle using a large number of evenly and closely placed loudspeakers. Ambisonics [5], which is another physically motivated approach to soundfield reproduction, is based on the first order spherical harmonic analysis of the soundfield to be reproduced. To achieve a large listening area, high order spherical harmonics have been introduced in Higher Order Ambisonics (HOA) [6]-[9], which is also widely used in multi-zone soundfield reproduction systems [10]-[12]. The reproduction performance of these approaches is satisfactory if the geometry of loudspeaker arrays used for reproduction is spherical [6], [13], [14] or circular [15], [16]. However, the performance degrades when the geometry becomes irregular. An improved least squares method with a weighted penalty function was developed for such irregular loudspeaker layouts [17]. This method requires the regularization parameter to be dependent on each loudspeaker arrangement.

On the other hand, perceptually motivated techniques aim to render the perceptually relevant aspects of the original sound. For example, there are binaural techniques [18], which provide a convincing experience over two channels. A multichannel perceptually motivated system, Vector Base Amplitude Panning (VBAP) [19]-[21], is designed to recreate the sound image of a virtual source over flexible loudspeaker layouts. VBAP is based on a triplet-wise panning law and the virtual source cannot be positioned outside a specific region, which decreases its applicability. Sena et al. recently developed a perceptual soundfield reconstruction system [22], which captures the directional cues by a designed microphone array, and reconstructs them using a loudspeaker array in the same configuration as the microphone array. Since Gerzon developed velocity and sound intensity theories of sound localization for reproducing psychoacoustically optimum sound [23], acoustic quantities containing directivity information, such as particle velocity and sound intensity, have been controlled in soundfield reproduction systems to improve the performance of perceptual localization. In addition to good performance for regularly or evenly placed loudspeakers [24] [27], the particle velocity or sound intensity based methods also perform well in irregular loudspeaker arrangements. A sound signal conversion method between different loudspeaker systems is proposed in [28], which reproduces the spatial impression of the original sound by maintaining the pressure and direction of sound (controlled by particle velocity). Shin et al. proposed a particle velocity controlled soundfield reproduction technique to achieve an improvement for human sound localization using non-uniformly spaced loudspeakers [29], 
[30]. However, the particle velocity is formulated analytically, implying that a practical system implementation requires to measure the particle velocity point by point. Furthermore, the work only focuses on particle velocity without sound pressure. In [31], authors introduced a soundfield reproduction method based on both sound pressure and particle velocity to improve the listening experience at a non-central listening point. The aforementioned works in particular have proven that particle velocity is an important predictor of perceived source directions, which is based on interaural phase cues and is most appropriate at frequencies below $700 \mathrm{~Hz}$ [23]. The sound intensity (i.e., the product of the sound pressure and particle velocity) is also a good predictor of localization perception [32]. It was exploited in the $\max -r_{E}$ decoding method [33], which is to reproduce the energy and acoustic intensity of the desired soundfield, and psychoacoustically to create the impression of the desired sound, especially for high frequencies (above $500 \mathrm{~Hz}$ ). In addition to accurate localization, the $\max -r_{E}$ decoding method can provide less coloration (i.e., changes in the timbre) compared with the pressure/particle velocity based methods [34]. By extending [33], the localization performance was improved by means of HOA [35]. However, the aforementioned works based on sound intensity are all restricted to a single reproduction position, and therefore perception degrades when the listener is moved from this exact reproduction position. In this paper, we propose to reproduce the original soundfield over a continuous spatial region rather than a single position by exploiting spatial intensity distributions.

We have recently formulated spatial sound intensity in the spherical harmonic domain [36], which means we can represent sound intensity that contains both energy and directivity information over continuous spatial regions. Therefore, similar to pressure matching in the spherical harmonic domain, intensity matching can also be realized over continuous space instead of at a single point so that the original sound can be perceptually reproduced over a large region for more listeners. In this work, we reproduce the sound intensity inside a continuous spatial region by matching sound intensity on the surrounding contour of the region using an irregular loudspeaker array. The remainder of this paper is structured as follows. First a brief review of the conventional spatial soundfield reproduction method, pressure matching in the spherical harmonic domain, is outlined in Section II. In Section III, spatial sound intensity representations are given and the intensity matching method is introduced in detail. Section IV demonstrates the accuracy of the proposed method by comparing it with the pressure matching and the HOA max $_{-} r_{E}$ decoding through numerical simulations. Perceptual listening test results are presented in Section V. Finally, the work is concluded in Section VI.

\section{REVIEW OF PRESSURE MATCHING IN THE SPHERICAL HARMONIC DOMAIN}

As a starting point, we briefly outline the concept of the pressure matching method [6]. In the spherical harmonic domain, the sound pressure at any point $\boldsymbol{x}=(r, \theta, \phi)$ in a spherical region, due to a desired source outside the region, can be written as

$$
P_{d}(\boldsymbol{x}, k)=\sum_{n=0}^{N} \sum_{m=-n}^{n} \alpha_{n m}(k) j_{n}(k r) Y_{n m}(\theta, \phi),
$$

where $N=\lceil k e R / 2\rceil$ is the truncation limit of the soundfield orders [37], $R$ is the radius of the sperical region, $e$ is the base of natural logarithm, $k=2 \pi f / c$ is the wave number, $f$ is the frequency, $c$ is the speed of propagation, $\alpha_{n m}(k)$ denotes spherical harmonic coefficients of the desired sound pressure, $j_{n}(\cdot)$ is the $n^{\text {th }}$ order spherical Bessel function of the first kind, and $Y_{n m}(\theta, \phi)$ is the spherical harmonic of order $n$ and degree $m$.

The reproduced soundfield is generated by a set of $N_{L}$ loudspeakers located at $\boldsymbol{x}_{\ell}=\left(r_{\ell}, \theta_{\ell}, \phi_{\ell}\right)$ outside the spherical region, with $\ell=1, \ldots, N_{L}$. We assume that the loudspeakers used for reproduction are point sources. Therefore, the radiation characteristics of the $\ell^{\text {th }}$ loudspeaker is determined by the Green's function [38],

$$
g\left(\boldsymbol{x} \mid \boldsymbol{x}_{\ell}, k\right)=\frac{e^{i k\left\|\boldsymbol{x}-\boldsymbol{x}_{\ell}\right\|_{2}}}{4 \pi\left\|\boldsymbol{x}-\boldsymbol{x}_{\ell}\right\|_{2}}
$$

where $\|\cdot\|_{2}$ denotes the Euclidean norm. Equation (2) can be decomposed as [39]

$$
g\left(\boldsymbol{x} \mid \boldsymbol{x}_{\ell}, k\right)=\sum_{n=0}^{N} \sum_{m=-n}^{n} \beta_{n m}^{(\ell)}(k) j_{n}(k r) Y_{n m}(\theta, \phi),
$$

with

$$
\beta_{n m}^{(\ell)}(k)=i k h_{n}\left(k r_{\ell}\right) Y_{n m}^{*}\left(\theta_{\ell}, \phi_{\ell}\right),
$$

where $h_{n}(\cdot)$ is the $n^{\text {th }}$ order spherical Hankel function of the first kind and $*$ denotes complex conjugate. The reproduced sound pressure due to the loudspeaker array can be written as

$$
P_{a}(\boldsymbol{x}, k)=\sum_{\ell=0}^{N_{L}} w_{\ell}(k) g\left(\boldsymbol{x} \mid \boldsymbol{x}_{\ell}, k\right),
$$

where $w_{\ell}(k)$ is the driving signal applied to the $\ell^{\text {th }}$ loudspeaker.

Substituting (3) into (5), and then equating (5) with (1), we have

$$
\begin{aligned}
& \sum_{n=0}^{N} \sum_{m=-n}^{n} \alpha_{n m}(k) j_{n}(k r) Y_{n m}(\theta, \phi) \\
& =\sum_{n=0}^{N} \sum_{m=-n}^{n} \sum_{\ell=0}^{N_{L}} w_{\ell}(k) \beta_{n m}^{(\ell)}(k) j_{n}(k r) Y_{n m}(\theta, \phi) .
\end{aligned}
$$

Multiplying both sides in (6) by $Y_{\hat{n} \hat{m}}^{*}(\theta, \phi)$ and integrating them over the unit sphere with respect to $\theta$ and $\phi$, gives

$$
\alpha_{n m}(k)=\sum_{\ell=0}^{N_{L}} w_{\ell}(k) \beta_{n m}^{(\ell)}(k) .
$$

Rewriting (7) in matrix form as

$$
\alpha=\beta G,
$$




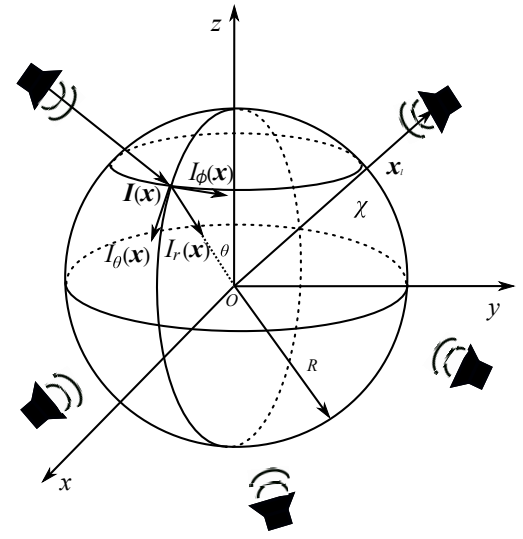

Fig. 1. Geometry of soundfield reproduction system in this paper. An irregular array of loudspeakers is located outside the spherical region.

where $\boldsymbol{\alpha}=\left[\alpha_{00}, \ldots, \alpha_{N N}\right]^{T}, \boldsymbol{G}=\left[w_{1}, \ldots, w_{N_{L}}\right]^{T}$, and

$$
\boldsymbol{\beta}=\left[\begin{array}{ccc}
\beta_{00}^{(1)} & \cdots & \beta_{00}^{\left(N_{L}\right)} \\
\beta_{1-1}^{(1)} & \cdots & \beta_{1-1}^{\left(N_{L}\right)} \\
\vdots & \ddots & \vdots \\
\beta_{N N}^{(1)} & \cdots & \beta_{N N}^{\left(N_{L}\right)}
\end{array}\right]
$$

For notations convenience, we omit the dependence on $k$. The number of loudspeakers $N_{L}$ specifies how the linear system (8) can be solved. There are three cases of interest. When the system is over-determined (i.e., $\left.(N+1)^{2}>N_{L}\right)$, there will be no exact solution in general. It becomes a least square problem

$$
\min _{\boldsymbol{G}}\|\boldsymbol{\beta} G-\boldsymbol{\alpha}\|_{2}^{2}
$$

If $\boldsymbol{\beta}$ is a square non-singular matrix, a unique solution to (8) is given by $\boldsymbol{G}=\boldsymbol{\beta}^{-1} \boldsymbol{\alpha}$. Finally, for an under-determined system (i.e., $\left.(N+1)^{2}<N_{L}\right)$, there may either be no solution or an infinite number of solutions. For the latter case, we can find the weights to satisfy

$$
\min _{\boldsymbol{G}}\|\boldsymbol{G}\|_{2}^{2} \text { s.t. } \quad \boldsymbol{\alpha}=\boldsymbol{\beta} \boldsymbol{G} .
$$

Tikhonov regularization can be introduced here to improve the robustness of the solution. The solutions of both the problems (10) and (11) are well studied in [40].

\section{INTENSITY BASED SPATIAL SOUNDFIELD REPRODUCTION}

In this section, we present the theory of intensity based spatial soundfield reproduction. The problem is formulated in Section III-A. After the spatial sound intensity representation in the spherical harmonic domain is given in III-B, how the loudspeaker weights are designed based on the representation is discussed in Section III-C.

\section{A. Problem formulation}

Consider a spherical target region $\chi$ of radius $R$ as seen in Fig. 1. Let there be an irregular array of loudspeakers outside of $\chi$, with the $\ell^{\text {th }}$ loudspeaker located at $\boldsymbol{x}_{\ell}$ with respect to the origin $O$. The free field assumption is made and diffraction/scattering is assumed to be negligible.
The complex acoustic intensity at any point $\boldsymbol{x}$ in $\chi$ can be represented by [41]

$$
\boldsymbol{I}(\boldsymbol{x}, k)=P^{*}(\boldsymbol{x}, k) \boldsymbol{V}(\boldsymbol{x}, k),
$$

where $\boldsymbol{I}(\boldsymbol{x}, k)=\left[I_{r}(\boldsymbol{x}, k), I_{\theta}(\boldsymbol{x}, k), I_{\phi}(\boldsymbol{x}, k)\right]$ and $\boldsymbol{V}(\boldsymbol{x}, k)=$ $\left[V_{r}(\boldsymbol{x}, k), V_{\theta}(\boldsymbol{x}, k), V_{\phi}(\boldsymbol{x}, k)\right]$ are sound intensity and particle velocity vector, respectively, $P(\boldsymbol{x}, k)$ is sound pressure. The real part of $\boldsymbol{I}(\boldsymbol{x}, k)$ is often referred to as the active sound intensity, which represents the propagating sound energy and shows the direction of propagation at the point in space. The imaginary part of it, on the other hand, is referred to as the reactive sound intensity, which represents the non-propagating sound energy [42].

A soundfield in a spherical region is usually characterized by spherical harmonic coefficients of sound pressure (which can be extracted by using higher order microphones such as an EigenMike) instead of the spatial distribution of sound intensity $^{1}$. Given spherical harmonic coefficients of sound pressure for the desired soundfield $\left\{\alpha_{n m}(k)\right\}$ in the spherical region $\chi$, and an appropriate irregular loudspeaker array geometry, our objective is to calculate the spatial sound intensity (12) in $\chi$ and find the loudspeaker driving signals that can reproduce the desired sound intensity over the continuous spatial region within $\chi$.

\section{B. Spatial sound intensity representation}

We have recently formulated spatial sound intensity in the spherical harmonic domain [36], which decomposes (12) in terms of spherical harmonic functions. We have shown that the spatial distribution of sound intensity can be expressed directly from the spherical harmonic coefficients of sound pressure.

Considering the spherical harmonic coefficients of sound pressure for the desired soundfield $\left\{\alpha_{n m}(k)\right\}$ are given, we can use the results of [36] to calculate the desired sound intensity at any point in $\chi$. According to the results in [36], [44], the components of desired sound intensity $\boldsymbol{I}^{d}(\boldsymbol{x}, k)=$ $\left[I_{r}^{d}(\boldsymbol{x}, k), I_{\theta}^{d}(\boldsymbol{x}, k), I_{\phi}^{d}(\boldsymbol{x}, k)\right]$ at any arbitrary $\boldsymbol{x}$ within $\chi$ can be decomposed, respectively, as

$$
\begin{aligned}
& I_{r}^{d}(\boldsymbol{x}, k)=\sum_{p=0}^{Q} \sum_{q=-p}^{p} S_{p q}^{(r, d)}(k, r) Y_{p q}(\theta, \phi), \\
& I_{\theta}^{d}(\boldsymbol{x}, k)=\sum_{p=0}^{Q} \sum_{q=-p}^{p} S_{p q}^{(\theta, d)}(k, r) Y_{p q}(\theta, \phi), \\
& I_{\phi}^{d}(\boldsymbol{x}, k)=\sum_{p=0}^{Q} \sum_{q=-p}^{p} S_{p q}^{(\phi, d)}(k, r) Y_{p q}(\theta, \phi),
\end{aligned}
$$

with

$$
\begin{aligned}
S_{p q}^{(r, d)}(k, r)= & \frac{i}{k \rho_{0} c} \sum_{n=0}^{N} \sum_{m=-n}^{n} \sum_{n^{\prime}=0}^{N} \sum_{m^{\prime}=-n^{\prime}}^{n^{\prime}}(-1)^{m+q} C_{n n^{\prime} p} \\
& \times \alpha_{n m}^{*}(k) \alpha_{n^{\prime} m^{\prime}}(k) j_{n}(k r) j_{n \prime}^{\prime}(k r) W_{1} W_{2},
\end{aligned}
$$

\footnotetext{
${ }^{1}$ The sound intensity can be estimated by measuring the particle velocity over the volume of interest point by point, which is time-consuming [43].
} 


$$
\begin{aligned}
& S_{p q}^{(\theta, d)}(k, r)=\frac{i}{k \rho_{0} c} \sum_{n=0}^{N} \sum_{m=-n}^{n} \sum_{n^{\prime}=0}^{N} \sum_{m^{\prime}=-n^{\prime}}^{n^{\prime}} A_{n m} A_{n^{\prime} m^{\prime}} A_{p q} \\
& \times \mathcal{P}_{n m n^{\prime} m^{\prime} p q} \mathcal{E}_{m m^{\prime} q} \alpha_{n m}^{*}(k) \alpha_{n^{\prime} m^{\prime}}(k) j_{n}(k r) j_{n \prime}(k r),
\end{aligned}
$$

and

$$
\begin{aligned}
& S_{p q}^{(\phi, d)}(k, r)=\frac{i}{k \rho_{0} c} \sum_{n=0}^{N} \sum_{m=-n}^{n} \sum_{n^{\prime}=0}^{N} \sum_{m^{\prime}=-n^{\prime}}^{n^{\prime}} i m^{\prime} A_{n m} A_{n^{\prime} m^{\prime}} \\
& \times A_{p q} \hat{\mathcal{P}}_{n m n^{\prime} m^{\prime} p q} \mathcal{E}_{m m^{\prime} q} \alpha_{n m}^{*}(k) \alpha_{n^{\prime} m^{\prime}}(k) j_{n}(k r) j_{n \prime}(k r)
\end{aligned}
$$

denoting spherical harmonic coefficients of desired sound intensity in $r, \theta$, and $\phi$ direction, respectively, where $C_{n n^{\prime} p}=$ $\sqrt{(2 n+1)\left(2 n^{\prime}+1\right)(2 p+1) / 4 \pi}, \quad A_{n m}=[(2 n+1)(n-$ $m) ! /(4 \pi(n+m) !)]^{1 / 2}, j_{n}^{\prime}(\cdot)$ is the derivative of $j_{n}(\cdot)$ in terms of $r, \rho_{0}$ is medium density,

$$
W_{1}=\left(\begin{array}{ccc}
n & n^{\prime} & p \\
0 & 0 & 0
\end{array}\right) \text {, and } W_{2}=\left(\begin{array}{ccc}
n & n^{\prime} & p \\
-m & m^{\prime} & -q
\end{array}\right)
$$

are Wigner 3-j symbols, $Q=2 N$ is truncation order for intensity expressions [36], and $\mathcal{E}_{m m^{\prime} q}=2 \pi$ when $m^{\prime}-m-q=0$, otherwise $\mathcal{E}_{m m^{\prime} q}=0$. Both $\mathcal{P}_{n m n^{\prime} m^{\prime} p q}$ and $\hat{\mathcal{P}}_{n m n^{\prime} m^{\prime} p q}$ are indices-only dependent variables, and they are given in our previous works [36], [44].

The intensity coefficients $S_{p q}^{(r, d)}(k, r), S_{p q}^{(\theta, d)}(k, r)$, and $S_{p q}^{(\phi, d)}(k, r)$ are obtained by decomposing sound pressure and particle velocity using spherical harmonic basis functions, and they represent the spatial intensity distribution of a soundfield. We note that the intensity coefficients are directly determined by the pressure coefficients $\alpha_{n m}(k)$ together with the radial functions (i.e., spherical Bessel function), and all the rest terms in the expressions are either constants or indices-only dependent variables. We select the same truncation order for all the components of sound intensity for simplicity in this work, at the cost of relatively more error on the components in $\theta$ and $\phi$ direction compared to $r$ direction.

\section{Loudspeaker weights design}

The loudspeakers are still modeled as point sources, and the spherical harmonic coefficients of sound pressure due to the $\ell^{\text {th }}$ loudspeaker in free field is given in (4). By replacing $\alpha_{n m}(k)$ with $\beta_{n m}^{(\ell)}(k)$ in (13) and (16), we obtain sound intensity in $r$ direction due to the $\ell^{\text {th }}$ loudspeaker at $\boldsymbol{x}$ as

$$
I_{r}^{(\ell)}(\boldsymbol{x}, k)=\sum_{p=0}^{Q} \sum_{q=-p}^{p} S_{p q}^{(r, \ell)}(k, r) Y_{p q}(\theta, \phi),
$$

where $S_{p q}^{(r, \ell)}(k, r)$ are spherical harmonic coefficients of sound intensity in $r$ direction due to the $\ell^{\text {th }}$ loudspeaker.

In order to drive loudspeakers, we apply a weight to each loudspeaker. Therefore, the total reproduced sound intensity in $r$ direction at $\boldsymbol{x}$ can be written as

$$
I_{r}^{a}(\boldsymbol{x}, k)=\sum_{p=0}^{Q} \sum_{q=-p}^{p} \underbrace{\sum_{\ell=0}^{N_{L}}\left|w_{\ell}(k)\right|^{2} S_{p q}^{(r, \ell)}(k, r)}_{S_{p q}^{(r, a)}(k, r)} Y_{p q}(\theta, \phi),
$$

where $N_{L}$ is the number of loudspeakers. We note that here we assume each loudspeaker is an independent source, and only time-average quantities (i.e., sound intensity) from them add up at the listeners' ears based on the fact that statistically out-of-phase sound signal summation occurs at the ears due to head diffraction [32], [45].

Similar to (20), the reproduced sound intensity in $\theta$ and $\phi$ directions at $\boldsymbol{x}$ can be expressed, respectively, as

$$
I_{\theta}^{a}(\boldsymbol{x}, k)=\sum_{p=0}^{Q} \sum_{q=-p}^{p} \underbrace{\sum_{\ell=0}^{N_{L}}\left|w_{\ell}(k)\right|^{2} S_{p q}^{(\theta, \ell)}(k, r)}_{S_{p q}^{(\theta, a)}(k, r)} Y_{p q}(\theta, \phi),
$$

and

$$
I_{\phi}^{a}(\boldsymbol{x}, k)=\sum_{p=0}^{Q} \sum_{q=-p}^{p} \underbrace{\sum_{\ell=0}^{N_{L}}\left|w_{\ell}(k)\right|^{2} S_{p q}^{(\phi, \ell)}(k, r)}_{S_{p q}^{(\phi, a)}(k, r)} Y_{p q}(\theta, \phi) .
$$

The reproduction problem is now reduced to calculate the loudspeaker driving signals/weights that can reconstruct the desired sound intensity over the target region. Inspired by Kirchhoff-Helmholtz integral equation, which shows that the soundfield inside a region can be controlled by evoking the sound pressure and the gradient of sound pressure on the surrounding contour of the region, we reproduce the sound intensity inside a spherical region by controlling the sound intensity (i.e., the product of sound pressure and its gradient) on the surface of the region ${ }^{2}$. Therefore, in this work, the problem is translated into minimizing the difference between the desired sound intensity and the reproduced sound intensity on the surface of the target region. The difference for sound intensity in $r$ direction can be given as

$$
E_{r}=\int_{0}^{2 \pi} \int_{-1}^{1}\left|I_{r}^{d}(R, \theta, \phi, k)-I_{r}^{a}(R, \theta, \phi, k)\right|^{2} d(\cos \theta) d \phi .
$$

Substitute (13) and (20) into (23), we have

$$
\begin{aligned}
E_{r} & =\sum_{p=0}^{Q} \sum_{q=-p}^{p} \sum_{p^{\prime}=0}^{Q} \sum_{q^{\prime}=-p^{\prime}}^{p^{\prime}}\left(S_{p q}^{(r, d)}(k, R)-S_{p q}^{(r, a)}(k, R)\right)^{*} \\
& \times\left(S_{p^{\prime} q^{\prime}}^{(r, d)}(k, R)-S_{p^{\prime} q^{\prime}}^{(r, a)}(k, R)\right) \\
& \times \int_{0}^{2 \pi} \int_{-1}^{1} Y_{p q}(\theta, \phi)^{*} Y_{p^{\prime} q^{\prime}}(\theta, \phi) d(\cos \theta) d \phi .
\end{aligned}
$$

Equation (24) can be simplified, by using the orthogonality of spherical harmonics, as

$$
E_{r}=\sum_{p=0}^{Q} \sum_{q=-p}^{p}\left|S_{p q}^{(r, d)}(k, R)-S_{p q}^{(r, a)}(k, R)\right|^{2} .
$$

Similarly, the difference for sound intensity in $\theta$ and $\phi$ direction are given, respectively, as

$$
E_{\theta}=\sum_{p=0}^{Q} \sum_{q=-p}^{p}\left|S_{p q}^{(\theta, d)}(k, R)-S_{p q}^{(\theta, a)}(k, R)\right|^{2},
$$

${ }^{2}$ While sound intensity matching on the surface of the target region is inspired by the Kirchhoff-Helmholtz principle, a formal proof showing that sound intensity matching on the boundary implies intensity matching within the volume is not currently available, and is a subject of future work. 
and

$$
E_{\phi}=\sum_{p=0}^{Q} \sum_{q=-p}^{p}\left|S_{p q}^{(\phi, d)}(k, R)-S_{p q}^{(\phi, a)}(k, R)\right|^{2} .
$$

A cost function containing all the components is given by

$$
f=c_{r} E_{r}+c_{\theta} E_{\theta}+c_{\phi} E_{\phi},
$$

where $c_{r}, c_{\theta}$, and $c_{\phi}$ are weighting coefficients. Different values of the weighting coefficients can be selected if different weights on the components are preferred. Also, one or two of the weighting coefficients can be set to zero (e.g., if one only focuses on the radial part of sound intensity, $c_{\theta}$ and $c_{\phi}$ can be set to zero). To minimize the cost function $f$, the problem can be formulated as

$$
\min _{\boldsymbol{W}}\left\|\boldsymbol{S}_{A} \boldsymbol{W}-\boldsymbol{S}_{D}\right\|_{2}^{2}, \quad \text { s.t. } \boldsymbol{W} \geq 0,
$$

where $\boldsymbol{S}_{D}=\left[\boldsymbol{S}^{(r, d)}(k)^{T}, \boldsymbol{S}^{(\theta, d)}(k)^{T}, \boldsymbol{S}^{(\phi, d)}(k)^{T}\right]^{T}$ is a $6(Q+$ $1)^{2}$ long vector with

$$
\begin{array}{r}
\boldsymbol{S}^{(O, d)}(k)=\sqrt{c_{O}}\left[\mathcal{R}\left\{S_{00}^{(O, d)}(k)\right\}, \mathcal{I}\left\{S_{00}^{(O, d)}(k)\right\}, \ldots,\right. \\
\left.\mathcal{R}\left\{S_{Q Q}^{(O, d)}(k)\right\}, \mathcal{I}\left\{S_{Q Q}^{(O, d)}(k)\right\}\right]^{T},
\end{array}
$$

where $\mathcal{R}\{\cdot\}$ denotes the real part, $\mathcal{I}\{\cdot\}$ denotes the imaginary part, $\boldsymbol{W}=\left[\left|w_{1}(k)\right|^{2},\left|w_{2}(k)\right|^{2}, \ldots,\left|w_{N_{L}}(k)\right|^{2}\right]^{T}$ is a $N_{L}$ long vector and $\boldsymbol{S}_{A}=\left[\boldsymbol{S}^{(r, a)}(k)^{T}, \boldsymbol{S}^{(\theta, a)}(k)^{T}, \boldsymbol{S}^{(\phi, a)}(k)^{T}\right]^{T}$ is a $6(Q+1)^{2}$ by $N_{L}$ matrix with

$$
\boldsymbol{S}^{(O, a)}(k)=\sqrt{c_{O}}\left[\begin{array}{ccc}
\mathcal{R}\left\{S_{00}^{(O, 1)}(k)\right\} & \cdots & \mathcal{R}\left\{S_{00}^{\left(O, N_{L}\right)}(k)\right\} \\
\mathcal{I}\left\{S_{00}^{(O, 1)}(k)\right\} & \cdots & \mathcal{I}\left\{S_{00}^{\left(O, N_{L}\right)}(k)\right\} \\
\vdots & \ddots & \vdots \\
\mathcal{R}\left\{S_{Q Q}^{(O, 1)}(k)\right\} & \cdots & \mathcal{R}\left\{S_{Q Q}^{\left(O, N_{L}\right)}(k)\right\} \\
\mathcal{I}\left\{S_{Q Q}^{(O, 1)}(k)\right\} & \cdots & \mathcal{I}\left\{S_{Q Q}^{\left(O, N_{L}\right)}(k)\right\}
\end{array}\right],
$$

where $O \in\{r, \theta, \phi\}$. $\boldsymbol{W} \geq 0$ means that each component of the vector $\boldsymbol{W}$ should be non-negative. Note that we deal with the real part and imaginary part of sound intensity separately as $\boldsymbol{W}$ is a real vector.

In mathematical optimization, this problem is known as non-negative least squares (NNLS). A widely used algorithm for solving this problem is the active-set method proposed by Lawson and Hanson in 1974 [46]. Other algorithms such as Landweber's gradient descent method [47] and coordinatewise optimization method [48] can also be applied to solving this problem. In this work, we solve (29) using Lawson and Hanson's method in [46]. We note that the weights obtained from the proposed method is real. To make the system causal and stable, a minimum-phase filter can be achieved from the real weight using a Hilbert transformer [49].

\section{Simulations}

The reproduction performance of the intensity matching, the pressure matching and the HOA $\max _{E} r_{E}$ decoding is now evaluated for a simulated free field environment, where the simulation setup and criteria are explained in Section IV-A. Afterward, a comparison for the intensity matching and the pressure matching is conducted in Section IV-B, and a
TABLE I

LOUDSPEAKER POSITIONS OF THE 5 CHANNEL SYSTEM

\begin{tabular}{cccc}
\hline \hline Loudspeaker No. & $r[\mathrm{~m}]$ & $\theta[\mathrm{deg}]$ & $\phi[\mathrm{deg}]$ \\
\hline 1 & 1 & 58.3 & 288 \\
2 & 1 & 58.3 & 72 \\
3 & 1 & 90 & 342 \\
4 & 1 & 90 & 234 \\
5 & 1 & 90 & 126 \\
\hline
\end{tabular}

TABLE II

LOUDSPEAKER POSITIONS OF THE 22 CHANNEL SYSTEM

\begin{tabular}{cccc}
\hline \hline Loudspeaker No. & $r[\mathrm{~m}]$ & $\theta[\mathrm{deg}]$ & $\phi[\mathrm{deg}]$ \\
\hline 1 & 0.97 & 0 & 0 \\
2 & 1.55 & 51.3 & 0 \\
3 & 1.55 & 51.3 & 45 \\
4 & 1.55 & 51.3 & 90 \\
5 & 1.55 & 51.3 & 135 \\
6 & 1.55 & 51.3 & 180 \\
7 & 1.55 & 51.3 & 225 \\
8 & 1.55 & 51.3 & 270 \\
9 & 1.55 & 51.3 & 315 \\
10 & 1.21 & 90 & 0 \\
11 & 1.21 & 90 & 22.5 \\
12 & 1.21 & 90 & 45 \\
13 & 1.21 & 90 & 90 \\
14 & 1.21 & 90 & 135 \\
15 & 1.21 & 90 & 180 \\
16 & 1.21 & 90 & 225 \\
17 & 1.21 & 90 & 270 \\
18 & 1.21 & 90 & 315 \\
19 & 1.21 & 90 & 337.5 \\
20 & 1.34 & 115 & 0 \\
21 & 1.34 & 115 & 45 \\
22 & 1.34 & 115 & 315 \\
\hline
\end{tabular}

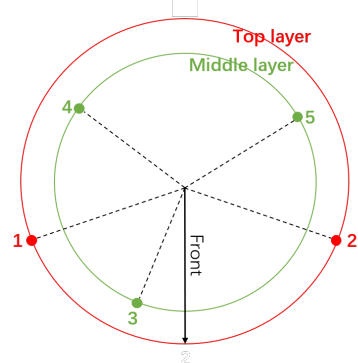

(a)

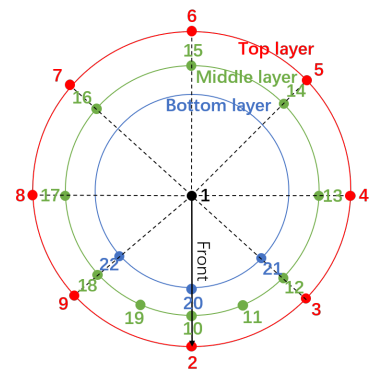

(b)
Fig. 2. 2D sketch of (a) the 5 channel system layout and (b) the 22 channel system layout.

comparison for the intensity matching and the HOA $\max -r_{E}$ decoding is shown in Section IV-C. Finally, it is investigated in Section IV-D how inherent noise of the microphones, which are required to measure the soundfield in practice, affects the reproduction performance.

\section{A. Simulation setup and criteria}

The target region is a spherical region $\chi$ with radius $R=$ $0.15 \mathrm{~m}$. To evaluate the overall performance of the proposed method, we simulate two different 3D loudspeaker layouts. One is composed of 5 separate loudspeakers and their positions are listed in Table I. Another loudspeaker layout is based on 


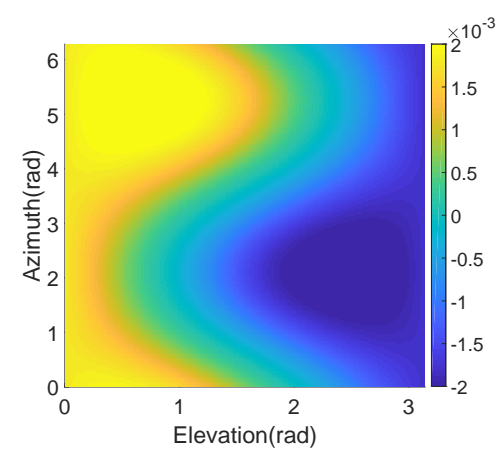

(a)

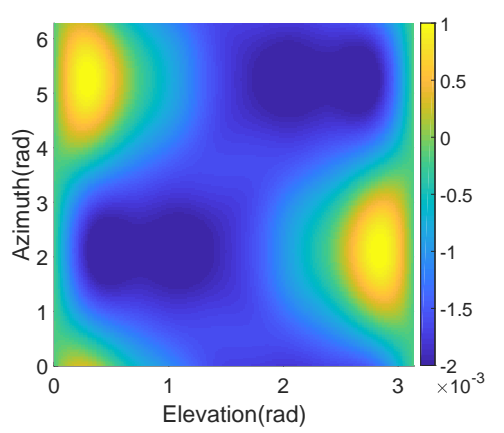

(b)

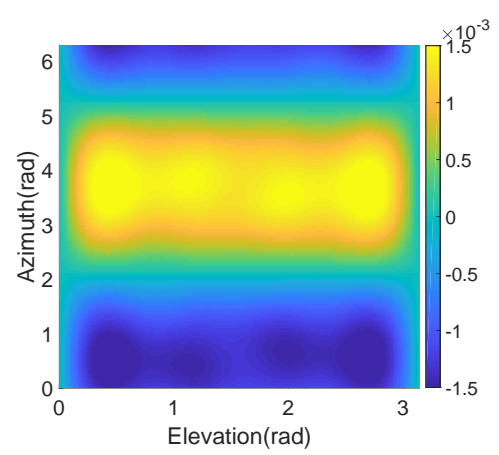

(c)

Fig. 3. Desired sound intensity in (a) $r$ direction, (b) $\theta$ direction, and (c) $\phi$ direction induced by a plane wave.

the cylindrical layout of 22.2 channel system, which is a wellknown 3D audio system, proposed by NHK [50] without the two low-frequency effect channels. The loudspeaker positions of the 22 channel system is given in Table II. A 2D sketch for both loudspeaker layouts is given in Fig. 2. We assume that the desired soundfield is a monochromatic plane wave. The spherical harmonic coefficients of sound pressure for the desired soundfield can be expressed as

$$
\alpha_{n m}=4 \pi(-i)^{n} Y_{n m}^{*}\left(\theta_{d}, \phi_{d}\right),
$$

where $\left(\theta_{d}, \phi_{d}\right)$ is the incident direction of the plane wave. Sound speed $c$ is $343 \mathrm{~m} / \mathrm{s}$ and air density is $1.29 \mathrm{~kg} / \mathrm{m}^{3}$. We set weighting coefficients $c_{r}=c_{\theta}=c_{\phi}=1$. As discussed in Section III-A, the active sound intensity represents the propagating sound energy and shows the propagation energy, therefore, we only evaluate the real part of the complex acoustic intensity in the following simulations.

As the first objective performance measure for the reproduction methods, the relative error $\epsilon$ of sound intensity is evaluated

$$
\epsilon(k)=10 \log _{10}\left(\frac{\sum_{\forall \hat{\boldsymbol{x}}}\left|\mathcal{R}\left\{I_{O}^{d}(\hat{\boldsymbol{x}}, k)-I_{O}^{a}(\hat{\boldsymbol{x}}, k)\right\}\right|^{2}}{\sum_{\forall \hat{\boldsymbol{x}}}\left|\mathcal{R}\left\{I_{O}^{d}(\hat{\boldsymbol{x}}, k)\right\}\right|^{2}}\right)(\mathrm{dB}),
$$

where $\hat{\boldsymbol{x}}$ denotes the evaluated point. To reproduce a soundfield with a realistic perception of the sound, it requires to ensure the reproduction of the direction of the sound. Therefore, the intensity direction error $\eta$ is also defined to show the difference in angles between two vectors

$$
\eta(\hat{\boldsymbol{x}}, k)=\cos ^{-1}(\mathrm{DOT}) / \pi \times 100(\%),
$$

where

$$
\operatorname{DOT}=\frac{\mathcal{R}\left\{\boldsymbol{I}^{a}(\hat{\boldsymbol{x}}, k)\right\}}{\left\|\mathcal{R}\left\{\boldsymbol{I}^{a}(\hat{\boldsymbol{x}}, k)\right\}\right\|_{2}} \cdot \frac{\mathcal{R}\left\{\boldsymbol{I}^{d}(\hat{\boldsymbol{x}}, k)\right\}}{\left\|\mathcal{R}\left\{\boldsymbol{I}^{d}(\hat{\boldsymbol{x}}, k)\right\}\right\|_{2}},
$$

with the desired intensity vector $\boldsymbol{I}^{d}(\hat{\boldsymbol{x}}, k)=$ $\left[I_{r}^{d}(\hat{\boldsymbol{x}}, k), I_{\theta}^{d}(\hat{\boldsymbol{x}}, k), I_{\phi}^{d}(\hat{\boldsymbol{x}}, k)\right]$ and the reproduced intensity vector $\boldsymbol{I}^{a}(\hat{\boldsymbol{x}}, k)=\left[I_{r}^{a}(\hat{\boldsymbol{x}}, k), I_{\theta}^{a}(\hat{\boldsymbol{x}}, k), I_{\phi}^{a}(\hat{\boldsymbol{x}}, k)\right]$, respectively, at $\hat{\boldsymbol{x}}$.

The following simulations study the performance of the proposed method, in terms of the reconstructed sound intensity, incident direction of the desired sound, frequency and reproduced sound direction. A plane wave with frequency of 900 $\mathrm{Hz}$ from $(2 \pi / 9,5 \pi / 3)$ is the desired soundfield throughout the simulations, except the evaluation in terms of the incident direction and frequency.

\section{B. Performance comparison for the intensity matching and the pressure matching}

1) Reproduced sound intensity: We first evaluate the reproduction of sound intensity on the surface of the target region. Figures 3(a), 3(b), and 3(c) show the desired sound intensity in $r, \theta$, and $\phi$ direction due to the plane wave, respectively. Figure 4 and Fig. 5 show the reconstructed sound intensity controlled by the intensity matching and the pressure matching using the 5 and 22 channel systems, respectively. Observe that, for the 5 channel system, the proposed method has much better performance than the pressure matching on the surface of the target region. As the number of channels increases, both of the methods perform better. However, the reconstructed sound intensity using the proposed method is still closer to the desired intensity than the pressure matching. The relative error on different spherical surfaces within the target region, which is calculated by (33), is also analyzed. The results are given in Fig. 6. For the 5 channel system, the relative error of the pressure matching for all the components of sound intensity is around $-5 \mathrm{~dB}$ in the target region, which is much larger than that of the intensity matching. That is because the matrix $\boldsymbol{\beta}$ in (8) for the 5 channel system is ill-conditioned. However, in this case, the intensity matching can provide a relatively accurate sound intensity reproduction with the error no more than $-10 \mathrm{~dB}$ within the target region. When more channels are available, the pressure matching performs better and the relative error for the whole region is around $-10 \mathrm{~dB}$. Meanwhile, the performance of the intensity matching is also improved, and the relative error is below $-25 \mathrm{~dB}$ within the target region. We note that, for both the 5 and 22 channel systems, the performance degrades as the radius increases because the active modes of spherical harmonics also increase when the radius increases. In this work, the system truncation limit is determined by the radius of the target region and the signal frequency, therefore, the error on the surrounding 


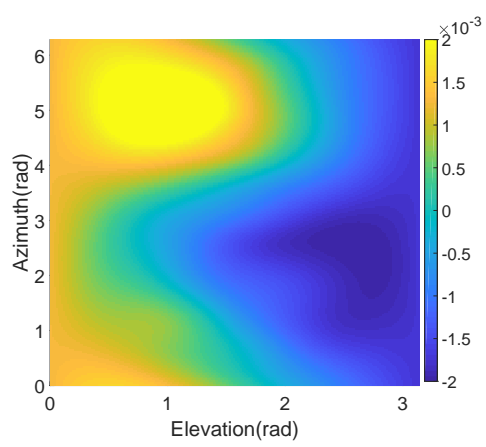

(a)

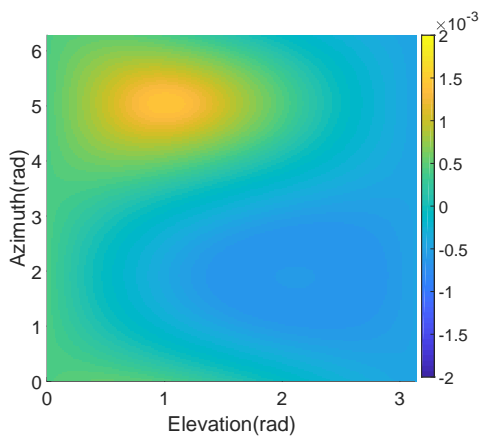

(d)

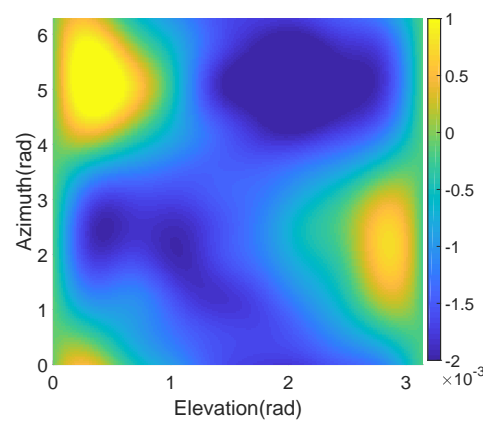

(b)

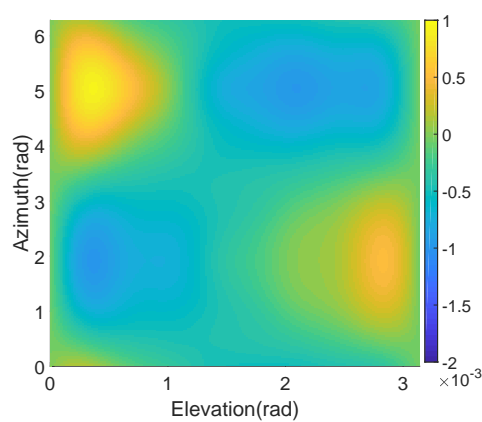

(e)

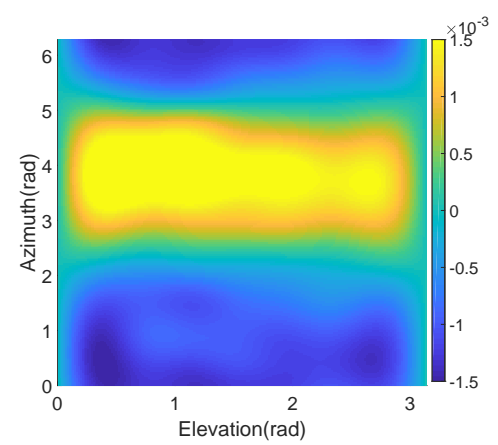

(c)

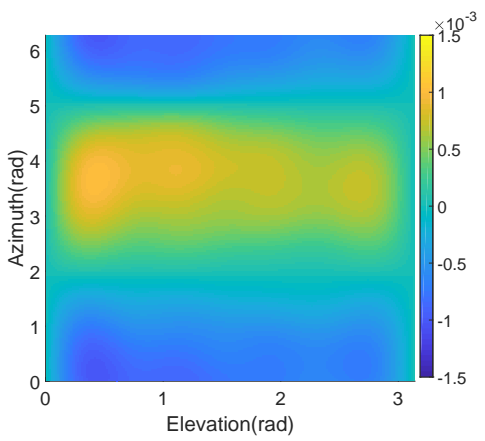

(f)

Fig. 4. Reconstructed sound intensity in (a, d) $r$ direction, (b, e) $\theta$ direction, and (c, f) $\phi$ direction controlled by (a-c) the proposed method, and (d-f) the pressure matching method in case of the 5 channel system.

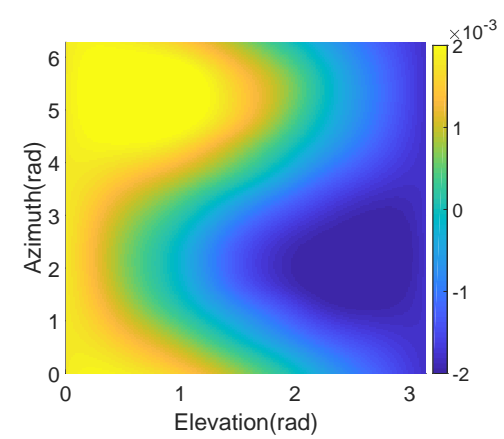

(a)

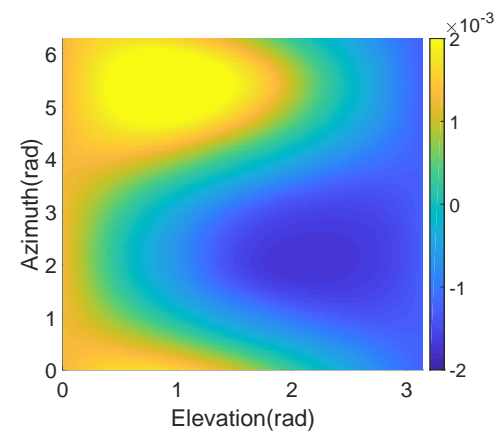

(d)

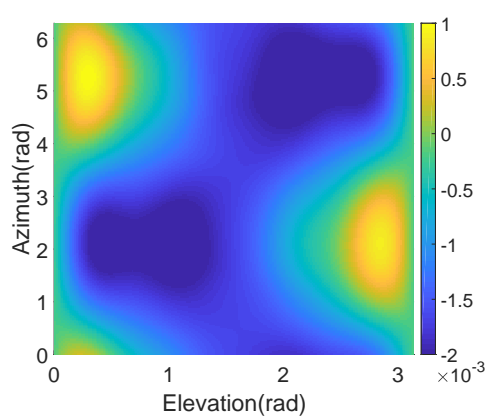

(b)

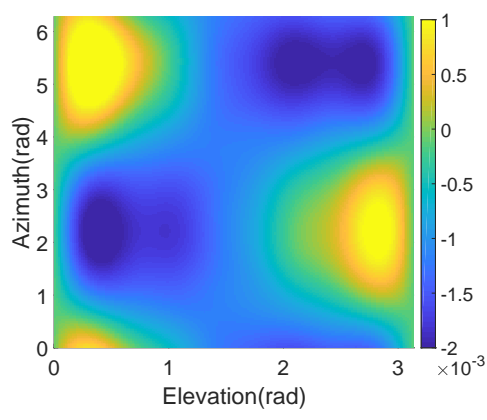

(e)

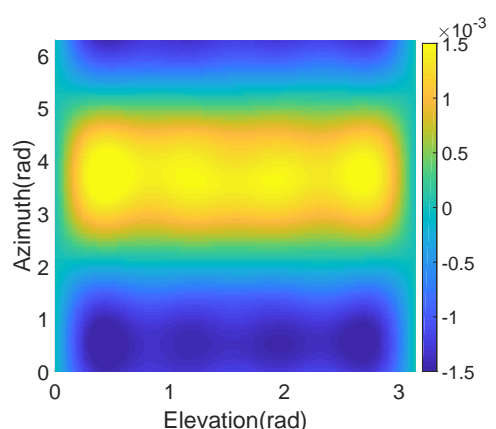

(c)

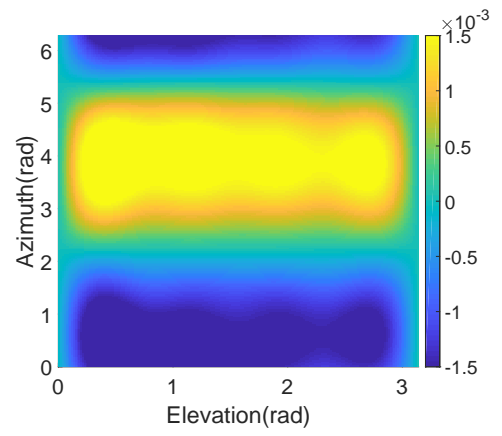

(f)

Fig. 5. Reconstructed sound intensity in (a, d) $r$ direction, $(\mathrm{b}, \mathrm{e}) \theta$ direction, and (c, f) $\phi$ direction controlled by (a-c) the proposed method, and (d-f) the pressure matching method in case of the 22 channel system.

contour is larger than that on the spherical surface inside the region due to the property of spherical Bessel function.
2) Incident direction of the desired sound: To investigate the influence on the performance of the two methods for 


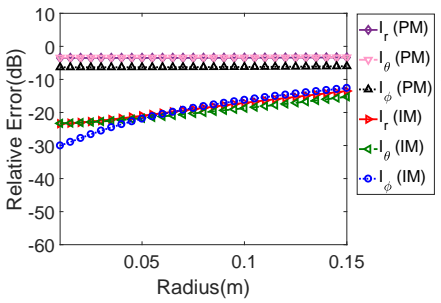

(a)

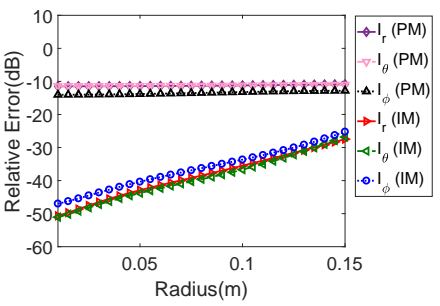

(b)
Fig. 6. Relative error with respect to the radius for (a) the 5 channel system and (b) the 22 channel system controlled by the intensity matching (IM) and the pressure matching (PM).

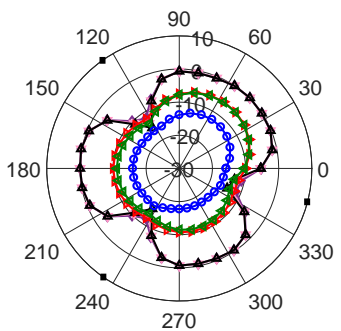

(a)
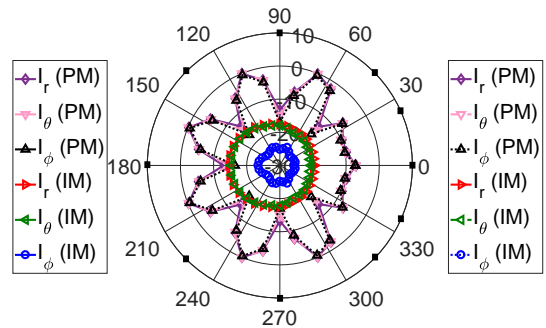

(b)
Fig. 7. Relative error on the surface of the target region with respect to the incident directions for (a) the 5 channel system and (b) the 22 channel system controlled by IM and PM. Symbol $\mathbf{\square}$ denotes the loudspeakers placed on the plane $z=0$.

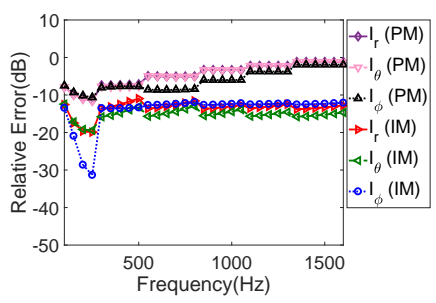

(a)

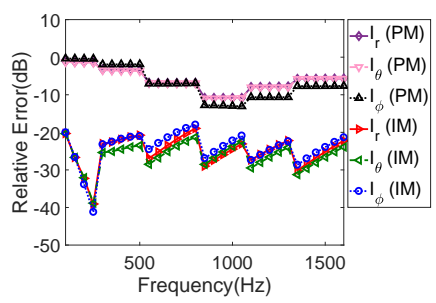

(b)
Fig. 8. Relative error on the surface of the target region with respect to the frequency for (a) the 5 channel system and (b) the 22 channel system controlled by IM and PM.

different incident directions of the desired soundfield, we consider all the incident directions on the plane $z=0$ (i.e., $\theta_{d}=\pi / 2$, and $\phi_{d}$ is from 0 to $\left.2 \pi\right)$. We choose the plane with the maximum error $(R=0.15 \mathrm{~m})$ as the observation sphere. Polar plots showing the relative error are given in Fig. 7, where symbol $\square$ denotes the loudspeakers placed on the plane $z=0$. From Fig. 7, it shows that the intensity matching and the pressure matching have similar performance when the incident direction is coincident with the location of one of the loudspeakers. Except for this case, the intensity matching performs better than the pressure matching at any other evaluated incident directions for both the loudspeaker layouts. An improvement of about $10 \mathrm{~dB}$ is obtained when the number of loudspeakers is increased from 5 to 22 . We note that it has relatively better performance for the directions where there are more loudspeakers for both the methods. For

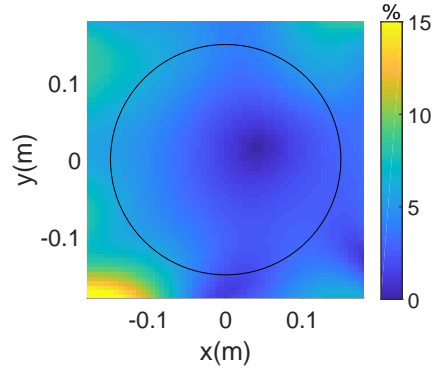

(a)

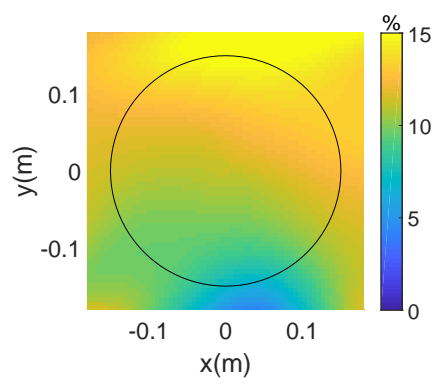

(c)

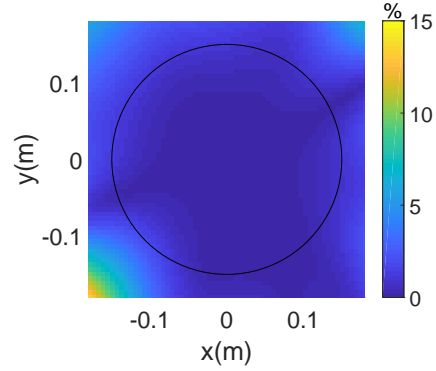

(b)

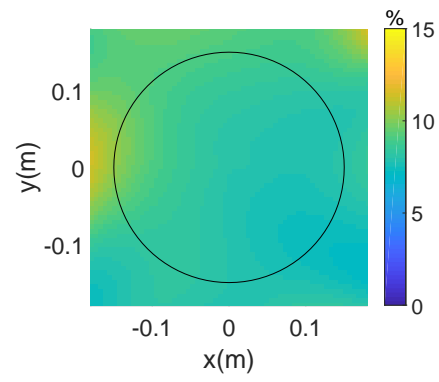

(d)
Fig. 9. The intensity direction error of $(a, c)$ the 5 channel system and $(b, d)$ the 22 channel system on plane $z=0$ controlled by (a-b) IM and (c-d) PM. Black circles denote the target region.

example, in Fig. 7(b), there are more loudspeakers located at the positions with $\phi_{d}$ from $0^{\circ}$ to $60^{\circ}$ and from $300^{\circ}$ to $360^{\circ}$, therefore, there is less error for the plane waves from these two direction intervals on plane $z=0$. An irregular loudspeaker array cannot well cover all the incident directions, which leads to relatively poor performance for the directions at which there are few loudspeakers. Moreover, the relative error of the pressure matching decreases sharply when the incident direction changes to be coincident with a loudspeaker location. For the intensity matching, it changes more smoothly, which can avoid the sudden deterioration of reproduction performance when the desired incident direction changes. Note that the patterns shown in Fig. 7 are dependent of the loudspeaker layouts.

3) Frequency: Figure 8 shows the relative error with respect to the change of frequency. The intensity matching is still better than the pressure matching within the frequency range evaluated for both the loudspeaker layouts. For the pressure matching, the system is either over-determined or underdetermined in the loudspeaker arrangements discussed in this work, which causes more error on the reproduction of sound intensity. However, the intensity matching optimizes the sound intensity directly using a cost function, which shows a better performance. Within a particular truncation limit $N$, similar to the increase of the radius, the relative error also increases as the signal frequency increases because of the property of spherical Bessel function. When the frequency increases to a value that makes the truncation limit become $N+1$, the relative error decreases compared to the error at the last frequency value, which makes the curves for the intensity matching sawtooth-shaped in Fig. 8. 


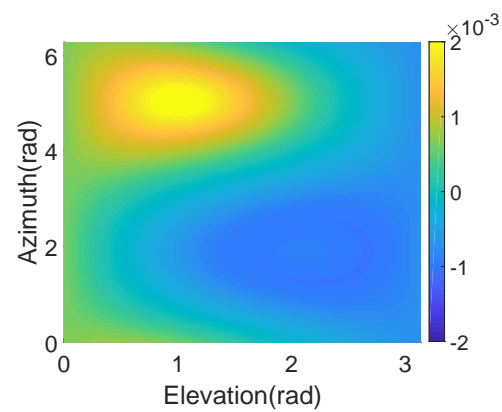

(a)

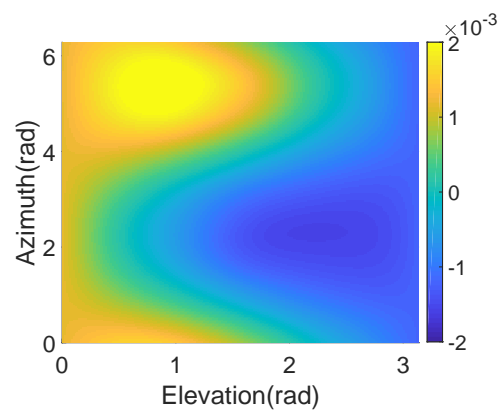

(d)

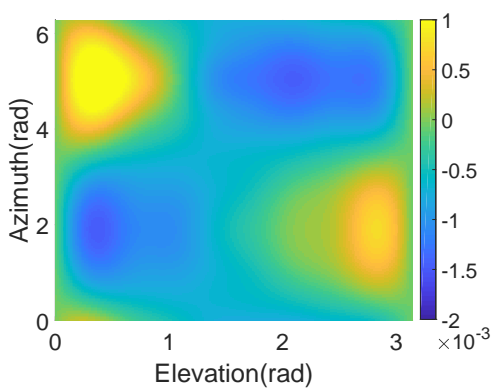

(b)

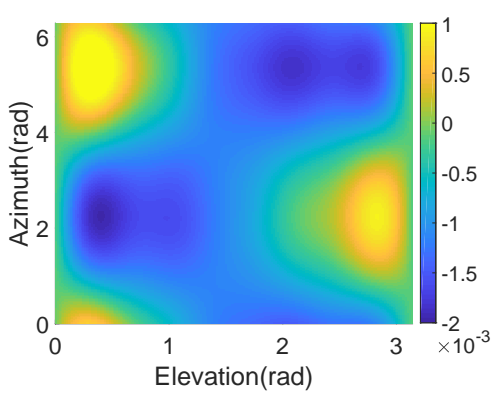

(e)

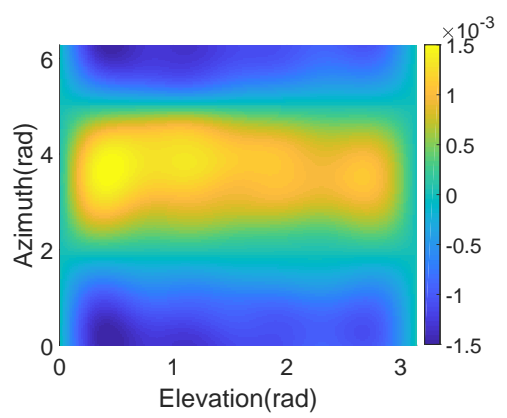

(c)

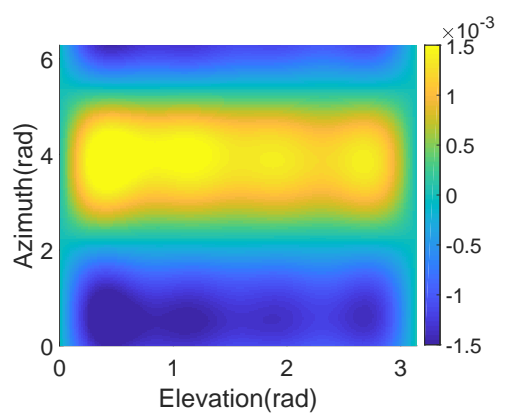

(f)

Fig. 10. Reconstructed sound intensity in (a, d) $r$ direction, (b, e) $\theta$ direction, and (c, f) $\phi$ direction controlled by the HOA max- $r_{E}$ decoding in case of (a-c) the 5 channel system, and (d-f) the 22 channel system.

4) Intensity direction: The discussion above has shown that all the components of sound intensity can be reproduced with limited error using the intensity matching. However, it is clear that if we are concerned to create a realistic perception of the original sound, it requires to ensure the reproduction of the direction of travel of the sound. Therefore, we calculate the intensity direction error on plane $z=0$ using (34), which is shown in Fig. 9. The black circles denote the target region on plane $z=0$. For the 5 channel system, the intensity direction error of the pressure matching is more than $10 \%$ for the whole region, whereas it is only approximately 5\% within the target region for the intensity matching. When the number of channels increases to 22 , the intensity direction error of the pressure matching is decreased to less than $10 \%$, and the performance of the intensity matching also becomes better, with the error around $2 \%$. It also shows that the intensity direction error inside the target region is less than that on the surrounding contour of the region for the intensity matching, which is consistent with the result of the relative error with respect to the radius.

\section{Performance comparison for the intensity matching and the HOA $\max -r_{E}$ decoding}

We also compare the proposed method with the HOA max $-r_{E}$ decoding [33], [35], which optimizes the energy and acoustic intensity at the origin. In the HOA $\max -r_{E}$ decoding, the driving signals fed to loudspeakers are given as

$$
G=D \alpha
$$

where $\boldsymbol{D}$ is the decoding matrix. The $\max -r_{E}$ decoding also assumes incoherent superposition of loudspeaker signals, and the loudspeakers are assumed as points sources. Therefore, the signal energy at the origin is given as

$$
E=\sum_{i=1}^{N_{L}} \frac{\left|w_{i}\right|^{2}}{\left(4 \pi d_{i}\right)^{2}},
$$

where $d_{i}$ is the distance from the $i^{\text {th }}$ loudspeaker to the origin. The normalized sound intensity at the origin can be written as

$$
\begin{aligned}
\boldsymbol{I} & =\frac{1}{E} \sum_{i=1}^{N_{L}} \frac{\left|w_{i}\right|^{2}}{\left(4 \pi d_{i}\right)^{2}} \hat{\boldsymbol{u}}_{i} \\
& =r_{E} \hat{\boldsymbol{v}}_{E},
\end{aligned}
$$

where $\hat{\boldsymbol{u}}_{i}$ is the unit vector corresponding to the position of the $i^{\text {th }}$ loudspeaker, $\hat{\boldsymbol{v}}_{E}$ is the unit vector representing the direction of the reproduced sound. This decoding is to maximize the value of $r_{E}$ with the condition that the direction of the reproduced sound is the direction of the desired sound. The cost function in [33] is used, without the energy and inphase components,

$$
f=\left(1-\boldsymbol{I} \cdot \hat{\boldsymbol{v}}_{j}\right)^{2}+\left(\left\|\boldsymbol{I} \times \hat{\boldsymbol{v}}_{j}\right\|\right)^{2},
$$

where $\hat{\boldsymbol{v}}_{j}$ is the unit vector representing the direction of the desired sound. We note that (39) is independent of frequency. This non-linear problem is solved by simulated annealing [33], which is a probabilistic technique, inspired from annealing in metallurgy, for approximating the global optimum of a given function.

1) Reproduced sound intensity: We also first evaluate the reproduction of sound intensity on the surface of the region. Fig. 10 shows the reconstructed sound intensity controlled by the HOA $\max _{E} r_{E}$ decoding for the 5 channel loudspeaker 


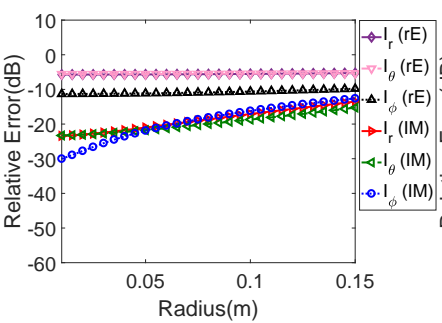

(a)

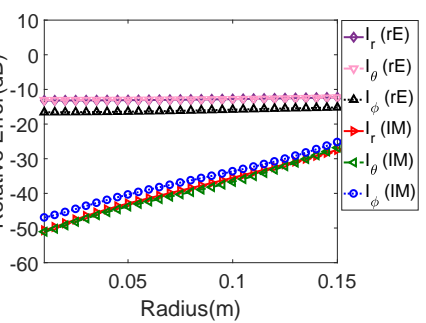

(b)
Fig. 11. Relative error with respect to the radius for (a) the 5 channel system and (b) the 22 channel system controlled by the intensity matching (IM) and the HOA $\max -r_{E}$ decoding $(\mathrm{rE})$.

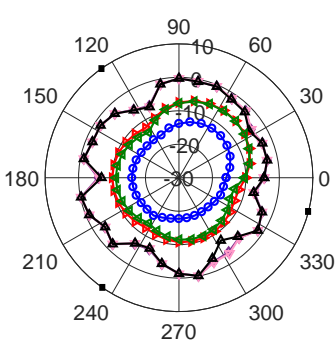

(a)
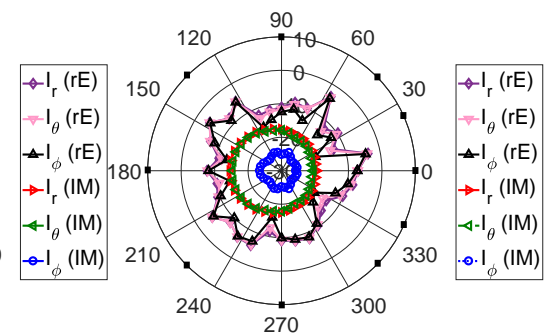

(b)
Fig. 12. Relative error on the surface of the target region with respect to the incident directions for (a) the 5 channel system and (b) the 22 channel system controlled by IM and rE. Symbol denotes the loudspeakers placed on the plane $z=0$.

layout and the 22 channel loudspeaker layout, respectively. It shows that the intensity matching has better performance than the HOA $\max _{E} r_{E}$ decoding for both loudspeaker geometries, by comparing the results with the reconstructed sound intensity controlled by the intensity matching. The relative error of sound intensity on different spherical surfaces within the target region is given in Fig. 11. We notice that the performance of the HOA $\max _{E} r_{E}$ decoding is also worse than that of the intensity matching, although it is slightly better than the pressure matching.

2) Incident direction of the desired sound: The same incident directions as in Section IV-B are evaluated for the HOA max $-r_{E}$ decoding, also compared with the intensity matching. The results are given in Fig. 12. In the 5 channel system, the intensity matching is approximately $10 \mathrm{~dB}$ better than the HOA $\max -r_{E}$ decoding for the evaluated incident directions. When the number of loudspeaker increases to 22, the performance of the HOA $\max _{E} r_{E}$ decoding also becomes better, however, it is not as good as the intensity matching. Different from the pressure matching, the HOA $\max r_{E}$ decoding does not have a significant improvement for the incident directions that are coincident with the location of one of the loudspeakers compared to other incident directions. The HOA $\max -r_{E}$ decoding has better performance than the pressure matching for most of the non-coincident directions. In terms of the smoothness of the error curves, the intensity matching also performs better than the HOA $\max _{E} r_{E}$ decoding.

3) Intensity direction: Intensity direction error of the HOA $\max _{E} r_{E}$ decoding is also calculated for both loudspeaker

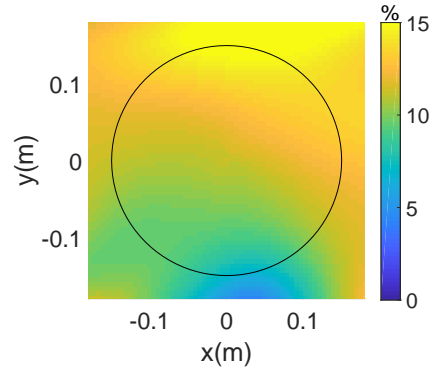

(a)

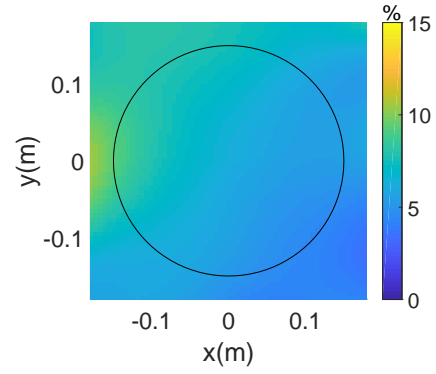

(b)
Fig. 13. The intensity direction error of (a) the 5 channel system and (b) the 22 channel system on plane $z=0$ controlled by the HOA $\max r_{E}$ decoding. Black circles denote the target region.

layouts, which is shown in Fig. 13. We compare the results with the intensity direction error of the intensity matching and the pressure matching correspondingly. For both loudspeaker geometries, the intensity matching is much better than the HOA $\max -r_{E}$ decoding. It is important to note that the performance of the HOA $\max _{E} r_{E}$ decoding is similar to the performance of the pressure matching for the 5 channel system. However, the HOA $\max r_{E}$ decoding is better than the pressure matching for the 22 channel system.

\section{Robustness analysis to microphone noise}

All above simulations are based on theoretical spherical harmonic coefficients of sound pressure of the desired soundfield. In practice, however, we calculate the coefficients based on sound pressure measured by a microphone array, which may introduce noise. To simulate this process, a spherical microphone array is considered and a White Gaussian Noise (WGN) with different signal to noise ratio (SNR) is added at each microphone. The truncation limit is $N=4$ for this case. Theoretically, a fourth order system should only require $(N+1)^{2}=25$ microphones on the surface of the target region. More accurate results are obtained, however, if more microphones are used. Therefore, we use a total of $(N+2)^{2}=36$ microphones placed on the surface of the 0.15 $\mathrm{m}$ sphere at location determined by [51]. The noisy pressure coefficients can be calculated by

$$
\hat{\alpha}_{n m}(k)=\frac{1}{j_{n}(k R)} \sum_{n_{M}=1}^{N_{M}} \hat{P}\left(r_{M}, \theta_{M}, \phi_{M}, k\right) Y_{n m}^{*}\left(\theta_{M}, \phi_{M}\right),
$$

where $\hat{P}\left(r_{M}, \theta_{M}, \phi_{M}, k\right)$ is the sound pressure measured at the $M^{\text {th }}$ microphone, and $N_{M}=36$ is the number of microphones. We first assess the proposed method by varying the SNR. The relative error is averaged across the entire target region and the results for both loudspeaker layouts are shown in Fig. 14. We note that the averaged relative error is less than $-10 \mathrm{~dB}$ for both loudspeaker layouts even though the SNR is as low as $10 \mathrm{~dB}$, and the average relative error decreases as the SNR increases. To compare the performance on different observation sphere for the noiseless and noisy conditions, we calculate the relative error by adding a WGN with SNR of 30 $\mathrm{dB}$ to the microphones. The results are given in Fig. 15. For 


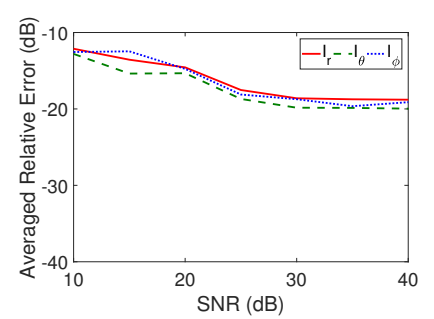

(a)

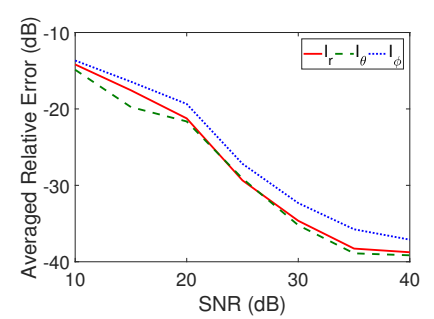

(b)
Fig. 14. Averaged relative error across the entire target region with respect to SNR for (a) the 5 channel system and (b) the 22 channel system.

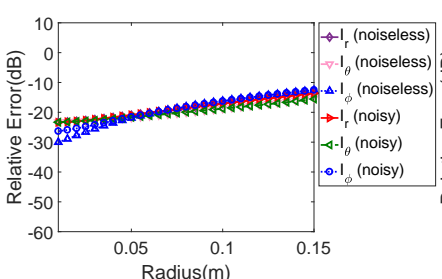

(a)

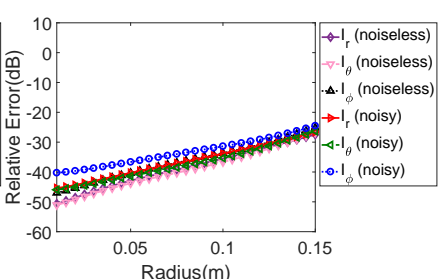

(b)
Fig. 15. Relative error with respect to the radius for (a) the 5 channel system and (b) the 22 channel system under the noiseless and noisy conditions.

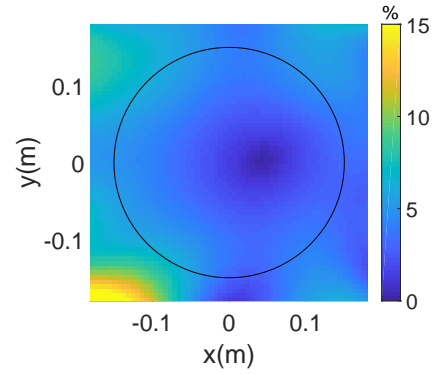

(a)

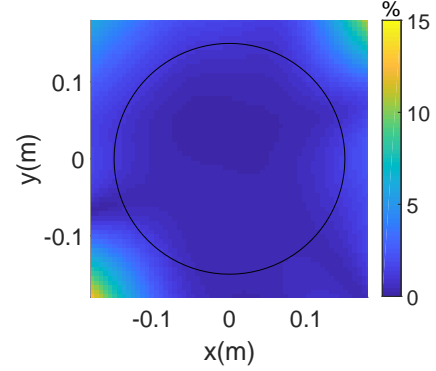

(b)
Fig. 16. The intensity direction error of (a) the 5 channel system and (b) the 22 channels when noise exists.

the 5 channel system, the curves for the noiseless and noisy cases are nearly coincided with each other, which means 30 $\mathrm{dB}$ WGN has little influence on it. For the 22 channel system, there is $5 \mathrm{~dB}$ deterioration near the center of the region for all the components of sound intensity when noise is introduced, however, the deterioration becomes less as the radius of the observation sphere increases. We also calculate the intensity direction error, which is shown in Fig.16. By comparing them with Fig. 9(a) and Fig. 9(b), we observe that the intensity direction error does not change a lot when the noise with SNR of $30 \mathrm{~dB}$ exists. These results indicate that microphone noise is not a severe problem for the proposed method in practice. Note that the proposed method does not work any more if the energy of the noise is high enough (e.g., $0 \mathrm{~dB}$ ) to ruin the pressure measurements.

\section{PERCEPTUAL LOCALIZATION EXPERIMENTS}

The 5 channel system is built for perceptual experiments in this section. Section IV has shown the HOA $\max -r_{E}$ decoding

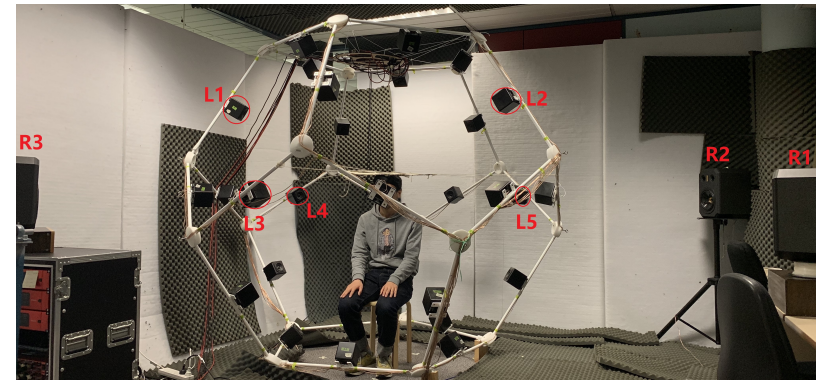

Fig. 17. Experimental setup for the 5 channel system.

TABLE III

THE LOCATIONS OF THREE DESIRED SOUND SOURCES

\begin{tabular}{cccc}
\hline \hline Label & $r[\mathrm{~m}]$ & $\theta[\mathrm{deg}]$ & $\phi[\mathrm{deg}]$ \\
\hline R1 & 3 & 90 & 60 \\
R2 & 3 & 90 & 126 \\
R3 & 3 & 90 & 306 \\
\hline \hline
\end{tabular}

TABLE IV

CMOS SCALE

\begin{tabular}{cc}
\hline \hline The direction of A compared to that of B is & Score \\
\hline much (more than $50^{\circ}$ ) closer to the reference & 3 \\
$\left(\right.$ more than $30^{\circ}$ ) closer to the reference & 2 \\
slightly (more than $10^{\circ}$ ) closer to the reference & 1 \\
(less than or equal to $10^{\circ}$ ) about the same & 0 \\
slightly (more than $10^{\circ}$ ) further to the reference & -1 \\
$\left(\right.$ more than $30^{\circ}$ ) further to the reference & -2 \\
much (more than $50^{\circ}$ ) further to the reference & -3 \\
\hline \hline
\end{tabular}

has similar performance to the pressure matching in the 5 channel system, therefore, we only assess the error between the actual directions and the perceived directions of sound sources as rendered by the intensity matching and the pressure matching in the experiments.

\section{A. Experimental setup and criteria}

The 5 channel system in Table I is built from a spherical loudspeaker array as shown in Fig 17, where only the five loudspeakers (circled and marked from L1 to L5) are activated and all the other loudspeakers are turned off. These five loudspeakers are used to play back stimuli generated by the intensity matching method and the pressure matching method. The center of the spherical array is set as the origin, and the direction where the listening subject faces is the positive $x$ axis. The target region $\chi$ is a spherical region centered by the origin with radius of $R=0.15 \mathrm{~m}$, which is large enough to fit a human head. There are another three loudspeakers (marked from R1 to R3) located outside of the spherical array, whose position is given in Table III. They serve as the desired sound sources/reference sources. All the loudspeakers are calibrated to produce the same sound pressure level within an error of $\pm 1 \mathrm{~dB}$. Sound absorbing materials are used in walls, floor and ceiling to reduce reflection. Diffraction/scattering is assumed to be negligible. This experiment is composed of three blind listening tests. For each test, the test samples consist of A, B and a reference, where the reference is the actual sound signal from the reference sources, and A and B are reproduced by the 


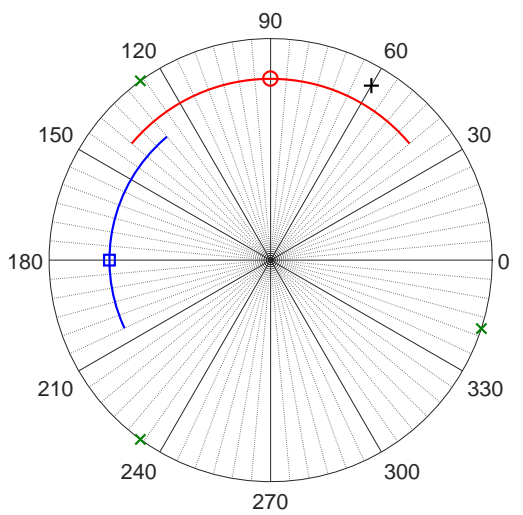

(a)

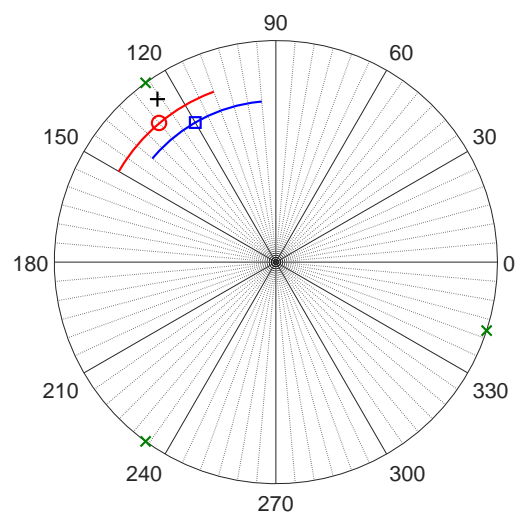

(b)

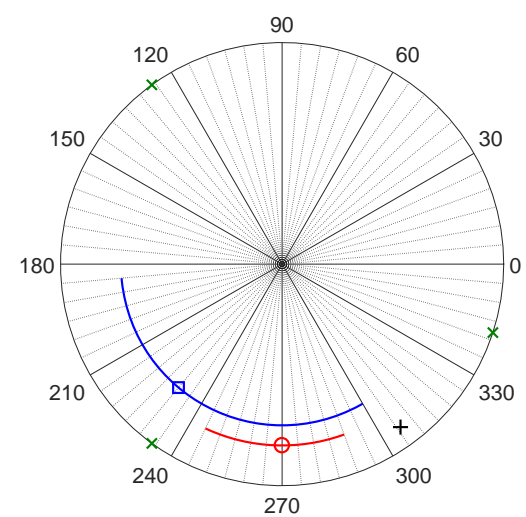

(c)

Fig. 18. Results of the perceptual localization experiments in the case of the 5 channel system controlled by the intensity matching (red lines with $\bigcirc$ ) and the pressure matching (blue lines with $\square$ ) for desired sound source (a) R1, (b) R2, and (c) R3. The symbols + denote the source positions and the symbols $\times$ denote the loudspeakers on the plane $z=0$ of the 5 channel system.

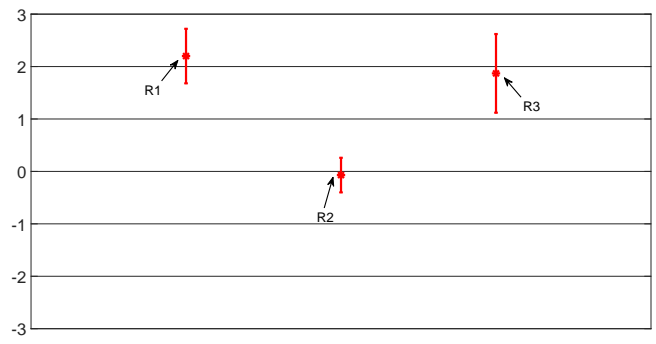

Fig. 19. CMOS scores for the three desired sources with $95 \%$ confidence intervals.

5 channel system using the intensity matching method and the pressure matching method, respectively. The hearing subjects do not have the knowledge of which method produces A, B, or the reference, and the hearing subjects are instructed to put their heads at the center and face the positive $x$ direction. During the period when the test samples are presented, the hearing subjects are allowed to slightly move their heads from the center. In each test, the hearing subjects are required to listen to all of the three test samples, and are given $10 \mathrm{~s}$ to record their perceived directions for each test sample from 72 angles $\left((\theta, \phi)=\left(90^{\circ}, 5^{\circ} \times(n-1)\right)\right.$, where $\left.n=1, \ldots, 72\right)$ on a provided answer sheet after listening to the samples. Each test sample is played twice, and there is a $2 \mathrm{~s}$ of pause between every two samples. A clean speech of $5 \mathrm{~s}$ duration is used as a source signal and the sample rate adopted is $48 \mathrm{kHz}$ for signal inputs to all of the loudspeakers. The reference is used not only to compare with $\mathrm{A}$ and $\mathrm{B}$, but also to identify an outlier within a data set (i.e., if the reference direction that a hearing subject perceives greatly deviates from its actual direction, the result from this subject is removed from the data set). We compare the perceived directions of $\mathrm{A}$ and $\mathrm{B}$ with the perceived direction of the reference, respectively, and evaluate the performance using Comparison Mean Opinion Score (CMOS). The score has 7 levels, which is shown in Table IV. The higher the CMOS score is, the better the intensity matching is versus the pressure matching.

\section{B. Experimental results}

17 subjects with normal hearing are recruited to participate in the listening tests. A valid data set containing 15 subjects is obtained after the outliers are removed. Figure 18 shows 10-90 percentile ranks that are described by lines for different desired sound sources. The symbols $(\bigcirc$ and $\square$ ) on the lines indicate the 50 percentile rank or median value. The red lines with $\bigcirc$ represent the subjective results of the intensity matching method, whereas the blue lines with $\square$ represent the subjective results of the pressure matching method. The percentile rank is used when there is a relatively small number of subjects because it does not consider any predefined distribution of the sample data set, which is regarded as a more representative expression [30]. As shown in Fig. 18(a), the test result controlled by the intensity matching shows much closer localization performance in terms of median value, although with slightly larger variations indicated by the angular range of the curve than the pressure matching when the desired sound source is located at position R1. In the case of desired source R2 in Fig. 18(b), which is coincident with the location of one of the loudspeakers in the 5 channel system, both the intensity matching and the pressure matching have good localization performance, which is consistent with the simulation results in Section IV-B. For desired source R3 in Fig. 18(c), the intensity matching provides more accurate localization performance with smaller variations than the pressure matching. Results in Fig. 18 also indicate that the proposed method can reproduce a strong direction perception of the desired sound in the case of there only being a small number of loudspeakers available.

We also calculate the CMOS scores for each desired source according to Table IV. The CMOS scores with $95 \%$ confidence intervals are given in Fig.19. A 95\% confidence interval is a range of values that you can be $95 \%$ certain contains the true mean of the population. The average score for desired source R2 is -0.07 , which means the direction reproduced by the intensity matching is about the same compared to that reproduced by the pressure matching. The average scores for desired sources R1 and R3 are 2.2 and 1.87, respectively, which means the intensity matching can reproduce a better 
original sound image compared to the pressure matching when the direction of desired sound is not coincident with the location of one of the loudspeakers for reproduction.

\section{CONCLUSION}

In this paper, we have studied the intensity matching technique used for soundfield reproduction over continuous space for an irregular loudspeaker array. The proposed method has an edge over the previous reproduction methods in the following aspects: i) a large and continuous listening area, ii) flexibility of the spatial loudspeaker arrangement, iii) a good sense of direction perceived by humans.

We represent sound intensity by spherical harmonic coefficients of sound pressure, and reproduce the soundfield inside the target region by controlling the sound intensity vectors on the surrounding contour of the region. To verify the performance of the proposed method, the intensity matching, as well as the pressure matching and the HOA $\max -r_{E}$ decoding, has been implemented in two loudspeaker arrangements, respectively, consisting of 5 and 22 non-uniformly placed loudspeakers in numerical simulations. The simulation results show that the proposed method can reproduce all the components of sound intensity and the direction of the desired sound inside the target region with less error than the pressure matching method and the HOA $\max -r_{E}$ decoding method. It is also proved that the proposed method has good robustness to microphone noise. Finally, the results of perceptual localization tests with the 5 channel system suggest that the proposed method can reproduce a better original sound image compared to the pressure matching with a small number of loudspeakers.

\section{REFERENCES}

[1] H. Hacihabiboglu, E. De Sena, Z. Cvetkovic, J. Johnston, and J. O. Smith III, "Perceptual spatial audio recording, simulation, and rendering: An overview of spatial-audio techniques based on psychoacoustics," IEEE Signal Process. Mag., vol. 34, no. 3, pp. 36-54, May 2017.

[2] A. J. Berkhout, D. de Vries, and P. Vogel, "Acoustic control by wave field synthesis," J. Acoust. Soc. Amer., vol. 93, no. 5, pp. 2764-2778, Jan. 1993.

[3] A. J. Berkhout, "A holographic approach to acoustic control," J. Audio Eng. Soc., vol. 36, no. 12, pp. 977-995, Dec. 1988.

[4] P.-A. Gauthier and A. Berry, "Adaptive wave field synthesis with independent radiation mode control for active sound field reproduction: Theory," J. Acoust. Soc. Amer., vol. 119, no. 5, pp. 2721-2737, Feb. 2006.

[5] M. A. Gerzon, "Ambisonics in multichannel broadcasting and video," $J$. Audio Eng. Soc., vol. 33, no. 11, pp. 859-871, Nov. 1985.

[6] D. B. Ward and T. D. Abhayapala, "Reproduction of a plane-wave sound field using an array of loudspeakers," IEEE Trans. Speech Audio Process., vol. 9, no. 6, pp. 697-707, Sept. 2001.

[7] T. Betlehem and T. D. Abhayapala, "Theory and design of sound field reproduction in reverberant rooms," J. Acoust. Soc. Amer, vol. 117, no. 4, pp. 2100-2111, Jan. 2005.

[8] M. A. Poletti, "Three-dimensional surround sound systems based on spherical harmonics," J. Audio Eng. Soc., vol. 53, no. 11, pp. 1004-1025, Nov. 2005.

[9] J. Ahrens and S. Spors, "Analytical driving functions for higher order ambisonics," in Proc. IEEE Int. Conf. Acoust. Speech Signal Process. (ICASSP), 2008, pp. 373-376.

[10] Y. J. Wu and T. D. Abhayapala, "Spatial multizone soundfield reproduction," in Proc. IEEE Int. Conf. Acoust. Speech Signal Process. (ICASSP), 2009, pp. 93-96.

[11] W. Jin and W. B. Kleijn, "Theory and design of multizone soundfield reproduction using sparse methods," IEEE/ACM Trans. Audio Speech Lang. Process., vol. 23, no. 12, pp. 2343-2355, Dec. 2015.
[12] W. Zhang, T. D. Abhayapala, T. Betlehem, and F. M. Fazi, "Analysis and control of multi-zone sound field reproduction using modal-domain approach," J. Acoust. Soc. Amer, vol. 140, no. 3, pp. 2134-2144, Sept. 2016.

[13] Y. J. Wu and T. D. Abhayapala, "Theory and design of soundfield reproduction using continuous loudspeaker concept," IEEE Trans. Audio Speech Lang. Process., vol. 17, no. 1, pp. 107-116, Jan. 2009.

[14] M. Poletti, F. Fazi, and P. Nelson, "Sound-field reproduction systems using fixed-directivity loudspeakers," J. Acoust. Soc. Amer, vol. 127, no. 6, pp. 3590-3601, Apr. 2010.

[15] A. Gupta and T. D. Abhayapala, "Three-dimensional sound field reproduction using multiple circular loudspeaker arrays," IEEE Trans. Audio Speech Lang. Process., vol. 19, no. 5, pp. 1149-1159, Jul. 2011.

[16] P. Chen, P. N. Samarasinghe, and T. D. Abhayapala, "3d exterior soundfield reproduction using a planar loudspeaker array," in Proc. IEEE Int. Conf. Acoust. Speech Signal Process. (ICASSP), 2018, pp. 471-475.

[17] M. Poletti, "Robust two-dimensional surround sound reproduction for nonuniform loudspeaker layouts," J. Audio Eng. Soc., vol. 55, no. 7/8, pp. 598-610, Jul. 2007.

[18] H. Møller, "Fundamentals of binaural technology," Appl. Acoust., vol. 36, no. 3-4, pp. 171-218, Mar. 1992.

[19] V. Pulkki, "Virtual sound source positioning using vector base amplitude panning," J. Audio Eng. Soc., vol. 45, no. 6, pp. 456-466, Jun. 1997.

[20] V. Pulkki and M. Karjalainen, "Localization of amplitude-panned virtual sources i: stereophonic panning," J. Audio Eng. Soc., vol. 49, no. 9, pp. 739-752, Sept. 2001.

[21] V. Pulkki, "Localization of amplitude-panned virtual sources ii: Twoand three-dimensional panning," J. Audio Eng. Soc., vol. 49, no. 9, pp. 753-767, Sept. 2001.

[22] E. De Sena, H. Hacrhabiboğlu, and Z. Cvetković, "Analysis and design of multichannel systems for perceptual sound field reconstruction," IEEE Trans. Audio Speech Lang. Process., vol. 21, no. 8, pp. 1653-1665, Aug. 2013.

[23] M. A. Gerzon, "Optimum reproduction matrices for multispeaker stereo," J. Audio Eng. Soc., vol. 40, no. 7/8, pp. 571-589, Jul. 1992.

[24] H. Hacihabiboğlu and Z. Cvetković, "Panoramic recording and reproduction of multichannel audio using a circular microphone array," in Proc. IEEE Workshop Appl. Signal Process. Audio Acoust. (WASPAA), 2009, pp. 117-120.

[25] A. Ando and K. Hamasaki, "Sound intensity-based three-dimensional panning," in Proc. 126th Audio Eng. Soc. Conv., May 2009.

[26] M. Buerger, R. Maas, H. W. Löllmann, and W. Kellermann, "Multizone sound field synthesis based on the joint optimization of the sound pressure and particle velocity vector on closed contours," in Proc. IEEE Workshop Appl. Signal Process. Audio Acoust. (WASPAA), 2015, pp. 1-5.

[27] M. Buerger, C. Hofmann, and W. Kellermann, "Broadband multizone sound rendering by jointly optimizing the sound pressure and particle velocity," J. Acoust. Soc. Amer, vol. 143, no. 3, pp. 1477-1490, Feb. 2018.

[28] A. Ando, "Conversion of multichannel sound signal maintaining physical properties of sound in reproduced sound field," IEEE Trans. Audio Speech Lang. Process., vol. 19, no. 6, pp. 1467-1475, Aug. 2011.

[29] M. Shin, F. M. Fazi, P. A. Nelson, and J. Seo, "Control of velocity for sound field reproduction," in Proc. 52th Int. Audio Eng. Soc. Conf., 2013.

[30] M. Shin, P. A. Nelson, F. M. Fazi, and J. Seo, "Velocity controlled sound field reproduction by non-uniformly spaced loudspeakers," J. Sound Vib., vol. 370, pp. 444-464, Feb. 2016.

[31] S. Wang, R. Hu, S. Chen, X. Wang, B. Peng, Y. Yang, and W. Tu, "Sound physical property matching between non central listening point and central listening point for nhk 22.2 system reproduction," in Proc. IEEE Int. Conf. Acoust. Speech Signal Process. (ICASSP), 2017, pp. $436-$ 440.

[32] M. A. Gerzon, "General metatheory of auditory localisation," in Proc. 92nd Audio Eng. Soc. Conv., 1992.

[33] D. Arteaga, "An ambisonics decoder for irregular 3-d loudspeaker arrays," in Proc. 134th Audio Eng. Soc. Conv., May 2013.

[34] M. Frank, "Phantom sources using multiple loudspeakers in the horizontal plane," Ph.D. dissertation, University of Music and Performing Arts Graz, Austria, 2013.

[35] D. Scaini and D. Arteaga, "Decoding of higher order ambisonics to irregular periphonic loudspeaker arrays," in Proc. 55th Int. Audio Eng. Soc. Conf., 2014.

[36] H. Zuo, P. N. Samarasinghe, T. D. Abhayapala, and G. Dickins, "Spatial sound intensity vectors in spherical harmonic domain," J. Acoust. Soc. Amer, vol. 145, no. 2, pp. EL149-EL155, Jan. 2019. 
[37] H. Zuo, P. N. Samarasinghe, and T. D. Abhayapala, "Exterior-interior 3d sound field separation using a planar array of differential microphones," in Proc. Int. Workshop Acoust. Signal Enhance. (IWAENC), 2018, pp. 216-220.

[38] E. G. Williams, Fourier Acoustics: Sound Radiation and Nearfield Acoustical Holography. Elsevier, 1999.

[39] P. Samarasinghe, T. Abhayapala, M. Poletti, and T. Betlehem, "An efficient parameterization of the room transfer function," IEEE/ACM Trans. Audio Speech Lang. Process., vol. 23, no. 12, pp. 2217-2227, Dec. 2015.

[40] C. F. Van Loan and G. H. Golub, Matrix Computations. Johns Hopkins University Press, 1983

[41] J. A. Mann III, J. Tichy, and A. J. Romano, "Instantaneous and timeaveraged energy transfer in acoustic fields," J. Acoust. Soc. Amer., vol. 82, no. 1, pp. 17-30, Mar. 1987.

[42] H. Chen, T. D. Abhayapala, P. N. Samarasinghe, and W. Zhang, "Directto-reverberant energy ratio estimation using a first-order microphone," IEEE/ACM Trans. Audio Speech Lang. Process., vol. 25, no. 2, pp. 226237, Feb. 2017.

[43] F. Jacobsen, and H. E. de Bree, "A comparison of two different sound intensity measurement principles," J. Acoust. Soc. Amer, vol. 118, no. 3, pp. 1510-1517, Jun. 2005.

[44] H. Zuo, P. N. Samarasinghe, T. D. Abhayapala, and G. Dickins, "Erratum: Spatial sound intensity vectors in spherical harmonic domain [j. acoust. soc. am. 145 (2), el149-el155 (2019)]," J. Acoust. Soc. Amer., vol. 146 , no. 1 , pp. 164-165, Jun. 2019

[45] J. Daniel, J.-B. Rault, and J.-D. Polack, "Ambisonics encoding of other audio formats for multiple listening conditions," in Proc. 105th Audio Eng. Soc. Conv., 1998.

[46] C. L. Lawson and R. J. Hanson, Solving Least Squares Problems. Siam, 1995.

[47] B. Johansson, T. Elfving, V. Kozlov, Y. Censor, P.-E. Forssén, and G. Granlund, "The application of an oblique-projected landweber method to a model of supervised learning," Math. Comput. Model., vol. 43, no. 7-8, pp. 892-909, Apr. 2006

[48] V. Franc, V. Hlaváč, and M. Navara, "Sequential coordinate-wise algorithm for the non-negative least squares problem," in Proc. Int. Conf. Comput. Anal. Images Patt., 2005, pp. 407-414.

[49] M. Hayes, J. Lim, and A. Oppenheim, "Signal reconstruction from phase or magmitude," IEEE Trans. Acoust. Speech Signal Process., vol. 28, no. 6, pp. 672-680, Dec. 1980.

[50] K. Hamasaki, K. Hiyama, and R. Okumura, "The 22.2 multichannel sound system and its application," in Proc. 118th Audio Eng. Soc. Conv., May 2005.

[51] H. R. H. Sloane N. J. A. and S. W. D. et al., Spherical Codes, [Online]. Available: http://neilsloane.com/packings/.

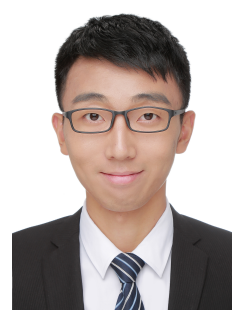

Huanyu Zuo received the Bachelor's degree and Master's degree in mechatronical engineering from the Beijing Institute of Technology, Beijing, China, in 2014 and 2017, respectively. He is currently working towards the Ph.D. degree in audio \& acoustic signal processing at the Australian National University (ANU), Canberra, Australia. His research interests include spatial sound recording and reproduction, 3D sound field separation, and psychoacoustics.

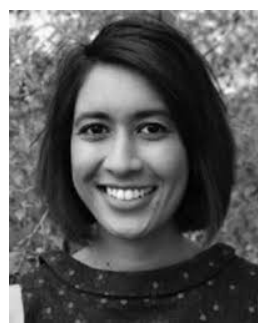

Prasanga N. Samarasinghe received the B.E. (Hons.) degree in electronic and electrical engineering from the University of Peradeniya, Peradeniya, Sri Lanka, in 2009, and the Ph.D. degree from the Australian National University (ANU), Canberra, ACT, Australia, in 2014. She is currently a Research Fellow with the Research School of Electrical, Energy and Materials Engineering, ANU. Her research interests include spatial sound recording and reproduction, spatial noise cancellation, and array optimization using compressive sensing techniques.

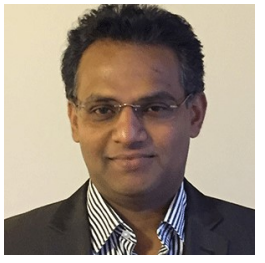

Thushara D. Abhayapala received the B.E. degree in engineering and the Ph.D. degree in telecommunications engineering from Australian National University (ANU), Canberra, ACT, Australia, in 1994 and 1999, respectively. He is a Professor of Signal Processing with ANU, Canberra, ACT, Australia. He held a number of leadership positions including Deputy Dean of the College of Engineering and Computer Science (20152019), Head of the Research School of Engineering with ANU (20102014) and the Leader of the Wireless Signal Processing Program at the National ICT Australia from 20052007. His research interests are in the areas of spatial audio and acoustic signal processing, and multichannel signal processing. Among many contributions, he is one of the first researchers to use spherical harmonic based eigen-decomposition in microphone arrays and to propose the concept of spherical microphone arrays; novel contributions on the multi-zone sound field reproduction problem; was one of the first to show the fundamental limits of spatial sound field reproduction using arrays of loudspeakers and spherical harmonics. He worked in industry for two years, before his doctoral study and has active collaboration with a number of companies. He has supervised $36 \mathrm{Ph} . \mathrm{D}$. students and coauthored more than 280 peer-reviewed papers. He was an Associate Editor for the IEEE/ACM TRANSACTIONS ON AUDIO, SPEECH, AND LANGUAGE PROCESSING and was a member of the Audio and Acoustic Signal Processing Technical Committee (20112016) of the IEEE Signal Processing Society. He is a fellow of Engineers Australia (IEAust). 